\title{
Effects of Aggregate Densification upon Thickening of Kynchian Suspensions
}

\author{
P. Grassia, ${ }^{\mathrm{a}, *}$, Y. Zhang ${ }^{\mathrm{a}}$, A. D. Martin ${ }^{\mathrm{a}}$, \\ S. P. Usher ${ }^{\mathrm{b}}$, P. J. Scales ${ }^{\mathrm{b}}$, A. H. Crust ${ }^{\mathrm{b}}$, R. Spehar ${ }^{\mathrm{b}}$ \\ ${ }^{a}$ CEAS, The Mill, University of Manchester, Oxford Road, Manchester M13 9PL, UK \\ ${ }^{b}$ Dept. Chemical \& Biomolecular Engineering, University of Melbourne, Parkville VIC 3010, Australia
}

\begin{abstract}
A model is presented for the thickening of a raked suspension. The model is based on Kynch theory (Kynch, 1952), i.e. it describes systems with solids fractions sufficiently low that the solids have not gelled into a weight-bearing network. However the model incorporates a modification to describe how raking the suspension causes flocs or aggregates within it to densify. This floc densification opens up channels between the flocs through which liquid escapes, making the suspension easier to dewater. The densification theory presented here predicts profiles of varying solids fraction vs height in the settling zone in a thickener, information which is not normally available when designing thickeners via conventional Kynch theory. Performance enhancements for thickeners due to raking can be readily determined, either in terms of increased underflow solids fraction or increased solids flux. As underflow solids fraction is increased, thickeners operated at a specified aggregate densification rate (or equivalently at a fixed settling zone height) tend to approach a 'fully densified' suspension state (defined as a point at which the extent of aggregate densification ceases to change with time), with improved thickening performance. Keywords:
\end{abstract}

Suspension, Rheology, Separations, Mathematical modelling, Thickening, Densification

\section{Highlights}

- Thickening of ungelled 'Kynchian' suspensions is considered

\footnotetext{
${ }^{*}$ Corresponding author

Email address: paul.grassia@manchester.ac.uk (P. Grassia)
} 
- Suspensions are raked causing flocs within them to densify

- Floc densification is predicted to enhance thickening performance

- Solids fraction profiles along a thickener can be computed even for Kynchian systems

- A fully densified state is approached as underflow solids fraction grows

\section{Introduction}

Dewatering of solid-liquid suspensions is a common engineering operation, with a multitude of applications, in areas including water recycling, waste management and minerals processing (Boger, 2009; Jones and Boger, 2012). There are many different types of engineering equipment available to achieve suspension dewatering, including batch settlers (Concha and Bustos, 1991), filter presses (Landman et al., 1991; Landman and White, 1994; Martin, 2004a), centrifuges (Berres et al., 2005a,b; Stickland et al., 2006), as well as continuous thickeners (Bürger et al., 1999; Bürger and Concha, 1998; Concha and Bustos, 1992; Doucet and Paradis, 2010; Landman et al., 1988; Martin, 2004b; Usher and Scales, 2005).

Chemical engineers are often faced with the task of designing and operating these types of equipment. However dewatering performance depends not solely upon the equipment specifications, but also upon the material properties of the suspension being dewatered (Landman and White, 1994). Accordingly there has been considerable effort in developing dewatering design methodologies that take explicit account of theories of suspension rheology, amongst the best known of them being that of Kynch (1952) and that of Buscall and White (1987).

These design methodologies tend to rely on phenomenological measurements of suspension rheology properties, for which measurement protocols already exist (Berres et al., 2005a,b; de Kretser et al., 2001; Green et al., 1998; Landman and White, 1992; Stickland et al., 2008). However the suspension rheology that is measured is strongly influenced by the suspension physical chemistry. Indeed in engineering practice, suspension dewatering rates are enhanced by addition of chemical flocculants (Usher et al., 2009). These chem- 
ical additives usually contain polymers which form bridges (Usher et al., 2009) from one individual solid particle to the next: the particle-polymer binding interactions tend to be sufficiently strong that the bonds can be considered to be irreversible (on time scales of interest). The solid particles joined by polymer bridges then form into a loose aggregate called a floc.

These aggregates or flocs tend to settle faster under the action of gravity than the individual solid particles. This is because when more and more individual particles are present in the floc, the downwards net buoyancy force grows more rapidly than its so called 'friction factor' (defined as the ratio between the frictional drag force on the floc and its speed relative to surrounding liquid). Indeed, whereas the buoyancy force is directly proportional to the number of individual solid particles in the floc (and hence to the overall floc volume), the friction factor tends to scale as the radius of the floc as a whole (Batchelor, 1967). This latter scaling follows because the surface area of the floc (over which frictional forces act) grows proportionally to the square of the floc radius, whilst the rate of strain (due to the floc moving with a given velocity relative to the surrounding liquid) scales inversely with the radius. It follows that if an aggregate or floc containing a given number of solid particles can be made more compact (such that its overall radius decreases) settling rates can be enhanced yet further.

One mechanism for achieving this is by raking the suspension, which can speed up dewatering rates in some cases by orders of magnitude (Gladman, 2006; Gladman et al., 2005, 2010; Usher et al., 2009; van Deventer et al., 2011). Raking in this fashion introduces shear forces that cause individual particles within an aggregate to displace relative to one another. Except in extreme cases where the imposed shear rates are so high as to tear aggregates apart altogether, these relative displacements produce additional contacts between particles and polymer bridges within the floc, binding the aggregate together rather more tightly (Mills et al., 1991; Usher et al., 2009; van Deventer et al., 2011). Here this process is called aggregate densification.

Aggregate densification is expected to affect the suspension material properties and thereby the dewatering performance. Indeed theories describing the effects of aggre- 
gate densification upon suspension material properties are already available in the literature (Usher et al., 2009; van Deventer et al., 2011; Zhang et al., 2013a,b). These theories determine material properties not only in terms of the solids fraction within the aggregates themselves, but also in terms of the overall solids fraction of the suspension (which takes account of zones of clear liquid, if any, between the flocs).

One key feature of the theories (Usher et al., 2009; Zhang et al., 2013a,b) is that significant benefits from aggregate densification are expected when overall solids fractions are 'low to moderate', which should be interpreted to mean that solids fraction should be low enough that flocs either remain isolated from one another (interacting with other flocs only hydrodynamically) or else are packed together only very loosely into an exceedingly fragile network. Under these circumstances, aggregate densification then opens up wide channels between flocs, through which liquid can escape. On the other hand, if the solids fraction in the suspension as a whole is comparatively high (e.g. comparable with the solids fraction in an individual floc), it follows that flocs are necessarily tightly packed together and must interpenetrate one another. There are no obvious preferred channels between the flocs for liquid escape, and so attempts to densify flocs may result in less dewatering benefit (Usher et al., 2009).

The aggregate densification theory has been used to model dewatering processes in batch settling (van Deventer et al., 2011) and also in the closely-related operation of continuous thickening (Usher et al., 2009; Zhang et al., 2013a,b). Here the focus is specifically upon on the case of buoyancy-driven thickening, where suspension is fed into and flows through the device, with concentrated suspension being drawn off as underflow at the bottom and with clear liquid being drawn off the top. Such a device combines the conventional chemical engineering benefits of a continuous operation, with the mathematical modelling simplicity for a device that (unlike a batch settler) can be made to operate at or near steady state. Indeed steady state operation of thickeners will be the focus in all that follows. Here 'steady state' refers to steady operation in the Eulerian sense. The thickener is of course unsteady in the Lagrangian sense: if one follows the settling solids, their state must be changing as dewatering occurs. 
It is noteworthy that the previously mentioned work on modelling thickening with aggregate densification (Usher et al., 2009; Zhang et al., 2013a,b) tended to focus on 'moderate to high' overall solids fractions. Specifically it focussed on cases where flocs were packed together into a network, possibly still with some gaps between flocs (moderate solids fraction) or else with flocs densely packed and interpenetrating (high solids fraction). With the exception of some of the cases considered by Usher et al. (2009), this previous work has not however considered thickening where the level of dewatering demanded is comparatively modest, in other words where the majority of the flocs within the thickener are fairly isolated from one another and so do not experience significant contact interactions with nearby flocs through e.g. being packed together into a network.

It might seem strange a priori that the previous modelling work on densified thickening considered primarily the high overall solids fraction regime (where the benefits of aggregate densification are in certain cases quite limited) and yet has seldom considered the regime of much lower target solids fractions (where the benefits of aggregate densification can be realised). The reason for this concerns the physical differences between low solids fraction suspensions and their moderate-to-high solids fraction counterparts, and how those physical differences consequently impact upon the resulting mathematical models.

Consider first of all, for simplicity, the nature of the theories in the absence of any aggregate densification. The particular overall solids fraction at which the flocs cease to be isolated, and instead form together into a network, is called the gel point (Landman and White, 1994). Suspensions at overall solids fractions below the gel point are typically modelled using Kynch theory (Kynch, 1952), which balances the buoyancy and viscous drag forces on a floc.

The key material property of the suspension that governs its dewatering behaviour is either a solids settling speed or a solids settling flux (functions of overall solids fraction (Kynch, 1952)), or a closely related property called the hindered settling factor (a measure of how the friction force grows with solids fraction due to solids interacting hydrodynamically (Landman and White, 1992), with direct contact interactions between solids being insignificant). Kynch theory applied to thickening (Concha and Bustos, 1992; Fitch, 
1966; Moncrieff, 1964; Shannon et al., 1963; Shannon and Tory, 1965; Shannon et al., 1964; Talmage and Fitch, 1955) enables one to investigate the relationship between the overall suspension solids fraction within the thickener, the suspension flux through the thickener and the solids fraction in the thickener underflow. This relationship is sensitive to the hindered settling induced by hydrodynamic interactions.

Suspensions at solids fractions above the gel point on the other hand are modelled using the theory of Buscall and White (1987), which incorporates an additional force (arising from a 'network stress gradient') in addition to buoyancy and viscous drag. The so called network stress (a measure of the weight-bearing strength of the network) is a material property that depends on the overall solids fraction. Thus the "network stress gradient' force in Buscall and White theory depends on the gradient of solids fraction. The theory therefore provides information about gradients of solids fraction, and hence about profiles of solids fraction versus position in the thickener. Thickener design computations using Buscall and White theory or analogous theories (Bürger et al., 1999; Bürger and Concha, 1998; Concha and Bustos, 1992; Landman et al., 1988; Martin, 2004b; Usher and Scales, 2005) are thereby more involved than those of Kynch theory, but the level of design detail extracted from Buscall and White theory is correspondingly greater.

The above discussion concerned thickener design for ungelled vs gelled suspensions in the absence of aggregate densification. If aggregate densification is now factored into the design computations in e.g. the case of a gelled suspension, thickening performance enhancements can be predicted (Usher et al., 2009; Zhang et al., 2013a,b) provided the floc network is not too densely packed together (to preclude any gaps whatsoever between the flocs), but the nature of the information that one extracts from a 'densified' Buscall and White theory is similar to what one obtains from the undensified theory.

In the case of an ungelled 'Kynchian' suspension however where the level of information that one extracts from the undensified theory is rather less, it might at first sight, appear that the resulting comparison between undensified and densified cases would be straightforward and perhaps even rather trivial. The purpose of this work is to demonstrate that even in the context of Kynch theory, thickener design calculations are not 
trivial in cases where aggregate densification is included. Indeed, on the contrary, a surprising amount of thickener design information can be readily extracted. In particular, one can deduce the detailed evolution of the state of the suspension as the solids migrate through the thickener: both the suspension solids fraction and the amount of aggregate densification evolve in a well defined way during the course of this migration. Since the state of the suspension defines the solids settling velocity, one can thereby relate the suspension's temporal evolution to a spatial profile through the thickener. This represents an advance over previous work, since even though Usher et al. (2009) did consider some cases of thickening of 'Kynchian' suspensions, it was assumed that flocs densified to a certain fixed level immediately upon entering the thickener, meaning that spatial information about the state of the suspension moving through the thickener were never deduced.

To summarise, there should be benefits (Arbuthnot et al., 2010; Loan and Arbuthnot, 2010a,b) from raking either a gelled or a Kynchian ungelled suspension (in the former case, provided the suspension solids fraction is not exceedingly far above the gel point). However the benefits deriving in the ungelled case are comparatively little explored, so that is the particular case considered here. Specifically, thickening performance enhancements will be computed and analysed for the case of an ungelled suspension in a thickener operated at steady state (in the Eulerian sense) whilst solid aggregates in suspension densify (in the Lagrangian sense) as they migrate through the system.

The rest of this work is laid out as follows. The models employed for thickening and aggregate densification are discussed in section 2, whilst section 3 briefly discusses numerical solution techniques for these models. Results of thickener design computations are presented in section 4 and discussion and conclusions are given in sections 5-6.

\section{Models for Kynchian Thickening and Aggregate Densification}

This section is laid out as follows. The section begins by reviewing a number of topics, namely Kynch theory (section 2.1), hindered settling factors (section 2.2), floc densification (section 2.3), the link between hindered settling and aggregate densification (sec-

tion 2.4), solids motion during thickening (section 2.5), and system non-dimensionalisation 
(section 2.6). The discussion proceeds to derive the novel densified thickener design equations that are required here, to describe respectively thickeners with specified suspension flux (section 2.7), thickeners with specified underflow solids fraction (section 2.8) and thickeners of specified height (section 2.9). The final topic addressed is preshearing suspensions prior to thickening (section 2.10).

\subsection{Review of Kynch theory}

The essential element of Kynch theory (Kynch, 1952) is a formula for the floc 'free settling' speed $u_{f s}$ as a function of the overall solids fraction $\phi$. This is written as:

$$
u_{f s}=u_{\text {Stokes }}(1-\phi)^{2} / r\left(\phi, \phi_{\text {agg }}\right)
$$

where $u_{\text {Stokes }}$ is the Stokes settling velocity of an isolated floc, $r\left(\phi, \phi_{\text {agg }}\right)$ in the denominator is the so called hindered settling factor (a specified function of both overall solids fraction $\phi$ and solids fraction within the aggregates $\left.\phi_{a g g}\right)$, and the $(1-\phi)^{2}$ factor in the numerator (Landman and White, 1994) comes from various effects (the settling velocity of a floc being slightly less than the slip velocity between a settling floc and surrounding liquid; as well as a tendency for the drag of the flocs upon the liquid to enhance the liquid pressure gradient, with this enhanced pressure then slightly slowing the flocs).

\subsection{Hindered settling factor}

The hindered settling factor $r$ will be considered as follows: first the hindered settling factor in the case of an undensified system is identified, and then (in a later section) there is a discussion of how to generalise to the case of a densified system.

Suppose that the aggregate solids fraction in the undensified system is denoted $\phi_{\text {agg, } 0}$, and that the hindered settling factor in the undensified system is denoted $r_{0}(\phi)$. Following previous work by Lester et al. (2005) it will be assumed that

$$
r\left(\phi, \phi_{a g g, 0}\right) \equiv r_{0}(\phi)=(1-\phi)^{-N}
$$

where $N$ is a parameter. That previous work (Lester et al., 2005) has assumed a com- 
paratively large value of the parameter $N$ (i.e. $N=20$ ), and the same value will be adopted here. Such a large $N$ value would indicate that the hydrodynamic interactions between flocs grow quite quickly with increasing $\phi$. Conceptually this follows because flocs are themselves (at least in the undensified state) quite loose structures. Hence the floc occupies a much larger volume (and so has a much larger hydrodynamic influence on neighbouring flocs) than in the hypothetical case where the same quantity of solids within a floc are closely packed with large spatial separation between neighbouring flocs.

\subsection{Review of floc densification}

Now consider the effects of densifying the floc. Following Usher et al. (2009) and van Deventer et al. (2011), it is assumed that under the action of shear, the diameter of the floc (relative to its initial diameter) denoted $D_{a g g}$ evolves with time $t$ (measured as time since material entered the shear field), as follows

$$
D_{\text {agg }}=\left(1-D_{\text {agg }, \infty}\right) \exp (-A t)+D_{a g g, \infty}
$$

Here $D_{a g g, \infty}$ is the final diameter ratio, i.e. the ratio between the diameter of a 'fully densified' floc and an undensified one. The fully densified state here represents a point at which the extent of aggregate densification ceases to change with time. In addition $A$ is an empirical rate parameter: for any given shear rate used to rake the suspension the value of $A$ can be readily measured. In the interests of simplicity we shall treat $A$ simply as a constant, although aggregate densification rate could in principle (van Deventer, 2012) be sensitive to system parameters such as solids fraction.

As the floc densifies, the solids fraction within it rises. Mass conservation of solids within the floc implies

$$
\phi_{\text {agg }} / \phi_{\text {agg }, 0}=D_{\text {agg }}^{-3}=\left(\left(1-D_{\text {agg }, \infty}\right) \exp (-A t)+D_{\text {agg }, \infty}\right)^{-3} .
$$

Throughout it will be assumed that $D_{a g g, \infty}=0.9$, a typical value employed in previous studies (Usher et al., 2009; van Deventer et al., 2011; Zhang et al., 2013a,b), although slightly lower values e.g. $D_{a g g, \infty}=0.846$ have also been observed experimentally (van 
Deventer et al., 2011). Clearly, based on equation (4), this chosen $D_{a g g, \infty}=0.9$ affects the possible values of the ratio $\phi_{\text {agg }} / \phi_{\text {agg, } 0}$, which consequently can never exceed 1.37. Meanwhile the value of $A$ in a 'typical' experiment (van Deventer et al., 2011) is $2 \times 10^{-3} \mathrm{~s}^{-1}$, although this is sensitive to the rate of raking, and values as high as $10^{-2} \mathrm{~s}^{-1}$ have been considered (van Deventer et al., 2011).

The theory presented below does not actually require the particular value of $\phi_{a g g, 0}$ to be specified: it merely requires that solids fraction $\phi$ be substantially smaller than $\phi_{a g g, 0} . \quad$ It is nevertheless instructive to estimate $\phi_{a g g, 0}$ as follows: Lester et al. (2005) quote a solids fraction of 0.22 as corresponding to the suspension gel point (without any aggregate densification). Meanwhile Usher et al. (2009) estimate that $\phi_{\text {agg }, 0}$ should be 1.67 times that, i.e. at solids fraction of around 0.37 (again without aggregate densification). Based on equation (4), as the system densifies, $\phi_{\text {agg }}$ could range as high as 0.50 . Different values of $\phi_{a g g 0}$ and/or $\phi_{a g g}$ are of course possible as a consequence of the vast range of particle size, shape and state of flocculation observed across a range of particulate suspensions (Usher et al., 2013) encountered in the field of dewatering.

\subsection{Effect of aggregate densification upon hindered settling}

The aggregate densification theory (Usher et al., 2009; van Deventer et al., 2011; Zhang et al., 2013a,b) allows one to express the hindered settling factor of a system of densified flocs in terms of that of an undensified system. The theory considers fluid flow both around and through flocs (Usher et al., 2009), but adopts a particularly simple form in the case considered here where the solids fractions under consideration are all relatively low (i.e. less than the system gel point). In that case, liquid flow around flocs completely dominates that through flocs (Zhang et al., 2013b). One then finds (see van Deventer et al. (2011); Zhang et al. (2013b) for the detailed explanation)

$$
r\left(\phi, \phi_{a g g}\right)=\frac{D_{a g g}(1-\phi)^{2} r_{0}\left(\phi_{a g g, 0} \phi / \phi_{a g g}\right)}{\left(1-\phi_{a g g, 0} \phi / \phi_{a g g}\right)^{2}}
$$

Since $r_{0}(\phi)$ tends to be a rapidly increasing function of its argument, it is clear that equation (5) for a sheared system predicts a much smaller value of $r$ than in the unsheared 
case of equation (2).

In equation (5), the parameter $\phi / \phi_{\text {agg }}$ represents the fraction of space filled by flocs. By substituting equation (5) into equation (1) one sees the batch settling speed of a system of densified flocs is virtually the same as that of a (distinct) system of undensified flocs, provided the fraction of space filled by flocs in the two systems is fixed.

There is however a small correction to this picture: in the $\phi \rightarrow 0$ limit, the settling speed of an isolated densified floc is a factor $D_{a g g}^{-1}$ greater than that of an isolated undensified floc. The reason is that the solids mass in a floc (and hence buoyancy force on the floc) remains fixed as it densifies, but its radius decreases, and hence the area over which frictional forces act decreases.

\subsection{Solids motion in a thickener}

The foregoing discussion concerned batch settling speeds. In a batch settler, solids flow downwards whilst liquid flows upward, but the net suspension flux (solids and liquid taken together) is nil. In the case of a thickener however, a net suspension flux flows $q$ out the bottom of the thickener. The solids velocity becomes

$$
\mathrm{d} z / \mathrm{d} t=-\left(q+u_{f s}\right)
$$

where the sign convention is such that distances $z$ are measured upwards, and velocities $q$ and/or $u_{f s}$ are measured downwards.

If the thickener settling zone height is $L$ and the total solids residence time is $t_{r e s}$ then it is clear that

$$
L=\int_{0}^{t_{r e s}}\left(q+u_{f s}\right) \mathrm{d} t
$$

where $u_{f s}$ at each time follows flocs as they settle and also densify in the thickener.

Since $u_{f s}$ is inversely proportional to $r$, and in particular, since $r$ is a rapidly decreasing function of solids fraction $\phi$, one can only use equation (7) to compute height of a thickener settling zone, if one knows the 'operating state' of the thickener i.e. the time history of $\phi$ values seen by a floc as it migrates through the thickener.

Finding the 'operating state' (in the presence of aggregate densification) turns out to 
be a generalisation of finding the 'operating point' (i.e. operating solids fraction) in a conventional (undensified) thickener, for which $u_{f s}$ becomes insensitive to residence time. The method for achieving this will be discussed shortly. First however the system of governing equations will be non-dimensionalised.

\subsection{Governing equations in dimensionless form}

It is convenient to make the system of equations dimensionless to facilitate the mathematical analysis. Velocities are made dimensionless with respect to $u_{\text {Stokes }}$ and hence it is possible to define $U_{f s}=u_{f s} / u_{\text {Stokes }}$ (dimensionless settling speed) and $Q=q / u_{\text {Stokes }}$ (dimensionless suspension flux). There are however two possible ways to make length and time scales dimensionless, and each of these is considered below.

\subsubsection{Scalings based on the densification rate parameter $A$}

Assuming that the densification rate parameter $A$ can be treated as a constant, one approach to non-dimensionalisation is to define $T=A t$ (dimensionless time), $Z=A z / u_{\text {Stokes }}$ (dimensionless distance). In this case, an important parameter is dimensionless total residence time $T_{\text {res }}$ in the shear field defined as $T_{\text {res }}=A t_{\text {res }}$, whilst the dimensionless height of the thickener settling zone is $l \equiv A L / u_{\text {Stokes. }}$. What these dimensionless scales imply in physical terms for a 'typical' suspension is described in the appendix.

For the aggregate diameter ratio and the solids fraction within the aggregates, the governing equations become respectively

$$
\begin{aligned}
D_{a g g} & =\left(1-D_{a g g, \infty}\right) \exp (-T)+D_{a g g, \infty} \\
\phi_{a g g} / \phi_{a g g, 0} & =D_{a g g}^{-3}=\left(\left(1-D_{a g g, \infty}\right) \exp (-T)+D_{a g g, \infty}\right)^{-3},
\end{aligned}
$$

while free settling speed is

$$
U_{f s}=(1-\phi)^{2} / r\left(\phi, \phi_{a g g}\right),
$$

the solids motion through the thickener is

$$
\mathrm{d} Z / \mathrm{d} T=-\left(Q+U_{f s}\right),
$$


and the dimensionless height satisfies

$$
l=\int_{0}^{T_{r e s}}\left(Q+U_{f s}\right) \mathrm{d} T
$$

\subsubsection{Scalings based on settling zone height $L$}

Alternatively it is possible to choose scalings such that $\tau=u_{\text {Stokes }} t / L$ (rescaled dimensionless time), $\zeta=z / L$ (rescaled dimensionless distance) and $\alpha=A L / u_{\text {Stokes }}($ dimensionless densification rate). In this case

$$
\begin{aligned}
D_{a g g} & =\left(1-D_{\text {agg }, \infty}\right) \exp (-\alpha \tau)+D_{\text {agg }, \infty} \\
\phi_{\text {agg }} / \phi_{\text {agg }, 0} & =D_{\text {agg }}^{-3}=\left(\left(1-D_{\text {agg }, \infty}\right) \exp (-\alpha \tau)+D_{\text {agg }, \infty}\right)^{-3} .
\end{aligned}
$$

The solids motion through the thickener is now

$$
\mathrm{d} \zeta / \mathrm{d} \tau=-\left(Q+U_{f s}\right)
$$

with the rescaled total residence time $\tau_{\text {res }}$ (which depends on $\alpha$ ) defined now as

$$
1=\int_{0}^{\tau_{r e s}}\left(Q+U_{f s}\right) \mathrm{d} \tau
$$

Bounds can be placed on the value of the integral on the right hand side (hence placing bounds on the possible values of the a priori unknown $\tau_{\text {res }}$ ) by evaluating the integrands either at an undensified operating point (slow densification limit), or at a fully densified operating point (rapid densification limit). Methods for determining operating points will be discussed shortly.

\subsubsection{Relation between the two formulations}

Observe that the dimensionless settling zone height $l$ in the first formulation (section 2.6.1) is actually numerically identical to the dimensionless densification rate parameter $\alpha$ in the second formulation (section 2.6.2): both are $A L / u_{\text {Stokes }}$. Here nonetheless, the two distinct symbols $l$ and $\alpha$ are retained as a reminder that physically, for a given 
suspension, there are two distinct ways to vary this parameter in experiments, i.e. varying settling zone height or varying raking rate.

Indeed the two formulations presented in sections 2.6.1-2.6.2 are physically equivalent, it merely being the case that in the first formulation one controls the value of residence time $T_{\text {res }}$ (so as to determine the height of the settling zone $l$ ) whilst in the second formulation one controls the value of the densification rate $\alpha$ (in order to determine the rescaled residence time $\left.\tau_{\text {res }}\right)$. Not only is it the case that $\alpha=l$ but also $\alpha \tau_{\text {res }}=T_{\text {res }}$, so the two different formulations simply swap the roles of the control and response variables. If one were to adopt the first formulation and vary the parameter $T_{\text {res }}$ until the computed settling zone height $l$ matched some required target value $\alpha$, that then is equivalent to the second formulation (a result that will be exploited later).

\subsection{Thickener design with a specified suspension flux}

There are various ways in which one can select the operating conditions of a thickener. If standard (undensified) Kynch theory is applied, typically one might specify the suspension flux $Q$, which is the case considered in this section. The theory (which permits discrete jumps in solids fraction) then predicts (Talmage and Fitch, 1955) one jump from at or around the feed solids fraction to some higher operating solids fraction corresponding to the hindered settling zone within the thickener, and then yet another jump in solids fraction at the underflow.

The most straightforward extension of this to the case of a densifying suspension, would specify the suspension flux $Q$ through the thickener and the total residence time $T_{\text {res }}$, interpreted for the purposes of the present discussion as the time to traverse the hindered settling zone. Instead of having a fixed solids fraction in the hindered settling zone, one now has a solids fraction that depends on time since entering that zone, i.e. $\phi=\phi(T)$ for $0 \leq T \leq T_{\text {res }}$, an 'operating state' rather than an 'operating point'. The techniques to identify and analyse that 'operating state' constitute the main novel contribution herein.

Observe that knowing $Q$ and $T_{\text {res }}$ is sufficient information to specify the solids flux $Q_{s}$ (as opposed to the suspension flux $Q$ ) through the thickener, and the underflow solids 
fraction $\phi_{u}$. Specifically (for a given feed solids fraction $\phi_{f}$ ) the solids flux is

$$
Q_{s}=\min _{\phi \geq \phi_{f}}\left(Q+U_{f s}\left(\phi, \phi_{\text {agg }}\left(T_{r e s}\right)\right) \phi\right.
$$

while the value of solids fraction at the bottom of the hindered settling zone (denoted now $\left.\phi\left(T_{r e s}\right)\right)$ is the specific value of $\phi$ corresponding to the minimum.

Typically one finds there is a critical value of $Q$ (the suspension flux) such that if the system is operated below that suspension flux, equation (17) possesses a local minimum for a $\phi$ value strictly greater than $\phi_{f}$. Operating the system at a suspension flux (and hence a solids flux) that is low enough that the solid flux curve possesses such a local minimum gives robustness in controlling and maintaining steady state thickening behaviour, in the face of e.g. fluctuations in solids fraction within the thickener.

Having identified $Q_{s}$, the solids fraction in the underflow $\phi_{u}$ becomes (Martin, 2004b)

$$
\phi_{u}=Q_{s} / Q
$$

This solids fraction (which always exceeds the solids fraction at the bottom of the settling zone $\left.\phi\left(T_{\text {res }}\right)\right)$ corresponds to a so called 'no slip' condition for the underflow (Martin, 2004b), i.e. solids and liquid are drawn off at the same speed there. Note that even though we have found $\phi\left(T_{r e s}\right)$ via Kynch theory, implying that it is a solids fraction below the suspension gel point, the somewhat larger value $\phi_{u}$ is (based on the 'no slip' condition) still permitted to be at or above the gel point.

Finally the profile of $\phi$ vs $T$ is obtained by specifying that the solids flux must be uniform through the hindered settling zone, so that

$$
\left(Q+U_{f s}\left(\phi(T), \phi_{\text {agg }}(T)\right) \phi(T)=\left(Q+U_{f s}\left(\phi\left(T_{\text {res }}\right), \phi_{\text {agg }}\left(T_{\text {res }}\right)\right) \phi\left(T_{\text {res }}\right) .\right.\right.
$$

As will become evident later (see e.g. the graphs plotted in Figure 1), this permits $\phi$ vs $T$ to be computed, and $\phi$ can increase with $T$ as one would expect to achieve dewatering. Equation (12) can now be used to determine the thickener height, whilst equation (11) can 
be used to convert the time history $\phi$ vs $T$ to a spatial profile $\phi$ vs $Z$. Note, by contrast, that conventional Kynch theory predicts a fixed value of $\phi$ in the settling zone (and also determines a much more dilute value of $\phi$, often of similar magnitude to the feed solids fraction $\phi_{f}$, that can co-exist with that in the settling zone through delivering the same solids flux): the conventional theory does not however predict where in the thickener the transition between these two states takes place. Profile information (i.e. predictions of how solids fraction varies with height) are not available via conventional Kynch theory without any aggregate densification.

\subsection{Thickener design with a specified underflow solids fraction}

An alternative way of specifying an undensified thickener is to give the underflow solids fraction. By extension, an alternate way of specifying a densified thickener is to give $\phi_{u}$ and $T_{\text {res }}$. In this case, the suspension flux in the thickener is (Usher and Scales, 2005)

$$
Q=\min _{\phi \geq \phi_{f}} U_{f s}\left(\phi, \phi_{\text {agg }}\left(T_{r e s}\right)\right) \phi /\left(\phi_{u}-\phi\right)
$$

with again $\phi\left(T_{\text {res }}\right)$ (solids fraction at the bottom of the hindered settling zone) being the value corresponding to the minimum. The value of solids flux $Q_{s}$ is $Q \phi_{u}$, and the profile of $\phi$ vs $T$ is computed as above via equation (19). Likewise thickener height $l$ and the profile $\phi$ vs $Z$ are computed via the techniques above.

\subsection{Thickener design with a specified settling zone height}

An alternative design specification is to give suspension flux $Q$ and settling zone height. When the system is specified in this way, it is simpler to use the alternative scalings (dimensionless spatial coordinate $\zeta$ and time $\tau$ in lieu of $Z$ and $T$ ). Equation (17) becomes

$$
Q_{s}=\min _{\phi \geq \phi_{f}}\left(Q+U_{f s}\left(\phi, \phi_{\text {agg }}\left(\tau_{\text {res }}\right)\right)\right) \phi
$$

with the solids fraction at the bottom of the hindered settling zone $\phi\left(\tau_{\text {res }}\right)$ corresponding to the minimum above. 
The difficulty however is that $\tau_{\text {res }}$ is not known a priori but must be obtained from equation (16) (and the value of $\tau_{\text {res }}$ that is computed is sensitive to the dimensionless densification rate parameter $\alpha$ ). It is expected that $\tau_{r e s}$ will be close to an undensified value (denoted $\tau_{\text {res }, 0}$ say) when $\alpha$ is small, and will migrate towards a smaller, fully densified value (denoted $\tau_{\text {res, } \infty}$ say) as $\alpha$ increases.

Once $\tau_{\text {res }}$ is available the solids fraction profile $\phi(\tau)$ can be obtained via

$$
\left(Q+U_{f s}\left(\phi(\tau), \phi_{\text {agg }}(\tau)\right)\right) \phi(\tau)=\left(Q+U_{f s}\left(\phi\left(\tau_{\text {res }}\right), \phi_{\text {agg }}\left(\tau_{\text {res }}\right)\right)\right) \phi\left(\tau_{\text {res }}\right)
$$

and the spatial profile $\phi$ vs $\zeta$ follows from equation (15).

Yet another alternative is to specify underflow solids fraction $\phi_{u}$ and thickener height. Analogously to equation (20) it is found that

$$
Q=\min _{\phi \geq \phi_{f}} U_{f s}\left(\phi, \phi_{\text {agg }}\left(\tau_{r e s}\right)\right) \phi /\left(\phi_{u}-\phi\right)
$$

with $\phi\left(\tau_{\text {res }}\right)$ (i.e. solids fraction at the bottom of the hindered settling zone) being given by the value corresponding to the minimum and with $Q_{s}=Q \phi_{u}$, but with $\tau_{\text {res }}$ still being a priori unknown and given by equation (16).

\subsection{Preshearing suspensions}

The above discussion (see equations (19) and/or (22)) considered the way in which a profile for solids fraction $\phi$ vs time $T$ (and hence a profile of $\phi$ vs position $Z$ ) can be obtained for $T$ values less than the total residence time $T_{\text {res }}$. It is not however a priori obvious that equations (19) and/or (22) necessarily admit solutions for all $T$ values $0 \leq T \leq T_{\text {res }}$. Indeed it turns out (as is actually apparent in Figure 1 to be discussed in detail later) that for sufficiently small $Q$, the equations admit solutions, but as $Q$ increases, it becomes increasingly difficult to satisfy them all the way down to $T=0$.

In that case, in order to exploit any operational benefits that aggregate densification is predicted to provide, it would become necessary to preshear the feed for some time $T_{\text {pre }}$ prior to it entering the thickener settling zone, a concept that has already been discussed by Zhang et al. (2013a). In other words, the material would need to be partially densified 
by shear even before entering the thickener. This may be costly to engineer (particularly if it were to involve retrofitting onto an existing thickener), and it is not clear that seeking to benefit from aggregate densification at a higher suspension flux $Q$ necessarily outweighs the potential costs: a simpler operational option in the high flux limit may be to forego preshear (and thereby forego any benefits that aggregate densification may bring), but this usually entails sacrificing the underflow solids fraction obtained. Assuming however that the engineering challenges associated with preshear are surmountable (perhaps e.g. via recycling material through the thickener (Doucet and Paradis, 2010; Schoenbrunn, 2011)), for the purposes of the discussion to follow, the symbol $T_{\text {res }}$ is used to denote the total time that the feed is subjected to the shear (comprised ${ }^{1}$ of preshear time $T_{\text {pre }}$ and time in the settling zone $\left.T_{\text {res }}-T_{\text {pre }}\right)$.

It should be emphasised that benefits of aggregate densification can always be realised without requiring preshear by choosing a small enough suspension flux $Q$. At larger $Q$ values, in order to benefit from aggregate densification, preshear may be required for sufficiently large $T_{\text {res }}$. Techniques for determining $T_{\text {pre }}$ in terms of $T_{\text {res }}$ are considered below. However given that preshear may be impractical or costly to achieve, any cases with nonzero $T_{\text {pre }}$ can be taken as indicative of $Q$ values for which the full benefits of aggregate densification cannot readily be realised.

\subsubsection{Systems with a specified total shearing time $T_{\text {res }}$}

To determine whether preshearing might be required (in order to obtain benefits from aggregate densification) at a given $T_{\text {res }}$ it is necessary to compare (a local maximum of) the undensified solids flux with (a local minimum of) the densified one: preshear should be considered if

$$
\max _{\phi \leq \phi\left(T_{r e s}\right)}\left(Q+U_{f s}\left(\phi, \phi_{a g g, 0}\right)\right) \phi<\min _{\phi \geq \phi_{f}}\left(Q+U_{f s}\left(\phi, \phi_{\text {agg }}\left(T_{r e s}\right)\right) \phi\right.
$$

\footnotetext{
${ }^{1}$ The definitions given here are more general than those used previously in section 2.7 , for which preshear was absent, meaning time in the settling zone equalled time subjected to shear.
} 
where on the left hand side of the inequality the symbol $\phi\left(T_{r e s}\right)$ is used to denote the value of $\phi$ found corresponding to the minimum on the right hand side.

In such cases the required amount of preshear time $T_{\text {pre }}$ turns out to satisfy

$$
\max _{\phi \leq \phi\left(T_{r e s}\right)}\left(Q+U_{f s}\left(\phi, \phi_{a g g}\left(T_{p r e}\right)\right) \phi=\min _{\phi \geq \phi_{f}}\left(Q+U_{f s}\left(\phi, \phi_{\text {agg }}\left(T_{r e s}\right)\right) \phi\right.\right.
$$

The height of the settling zone is now given by (in lieu of equation (12))

$$
l=\int_{T_{p r e}}^{T_{\text {res }}}\left(Q+U_{f s}\right) \mathrm{d} T .
$$

The above concerns the case where the residence time $T_{r e s}$ is specified and the height of the settling zone must be calculated. As has been discussed previously, an alternative way to formulate the problem is to fix the thickener height (i.e. the height of the settling zone) but to vary the rate of raking. This is considered in the next section.

\subsubsection{Systems with a specified densification rate $\alpha$}

There is now a control parameter $\alpha$ (the dimensionless densification rate) and one must now determine a (rescaled) residence time $\tau_{\text {res }}$ and if necessary a (rescaled) preshearing time $\tau_{\text {pre }}$ as follows.

First it is necessary to compare the undensified solids flux function with the fully densified one to establish whether

$$
\max _{\phi \leq \phi_{\infty}}\left(Q+U_{f s}\left(\phi, \phi_{\text {agg }, 0}\right)\right) \phi \geq \min _{\phi \geq \phi_{f}}\left(Q+U_{f s}\left(\phi, \phi_{\text {agg }, \infty}\right)\right) \phi
$$

where $\phi_{\infty}$ on the left hand side is the value of $\phi$ determined by the minimisation on the right, with $\phi_{a g g, \infty}$ being the aggregate solids fraction in the fully densified state. If this relation is satisfied, then preshearing is never required (at that given $Q$ ), i.e. thickening performance enhancements can be derived from aggregate densification even in the absence of preshear. If however

$$
\max _{\phi \leq \phi_{\infty}}\left(Q+U_{f s}\left(\phi, \phi_{a g g, 0}\right)\right) \phi<\min _{\phi \geq \phi_{f}}\left(Q+U_{f s}\left(\phi, \phi_{a g g, \infty}\right)\right) \phi
$$


which tends to occur for sufficiently large $Q$, then preshear might be needed in order to exploit the benefits of aggregate densification. Whether or not preshear is needed now depends on the value of the dimensionless densification rate $\alpha$ : high densification rates (i.e. high $\alpha$ ) require preshearing, whereas low densification rates (i.e. low $\alpha$ ) do not.

The symbol $\alpha_{\text {crit }}$ (a function of $Q$ ) can be used to denote the boundary between cases which involve preshear and those that do not, and $\tau_{\text {res, crit }}$ (again a function of $Q$ ) to denote the residence time that satisfies equation (16) for $\alpha=\alpha_{\text {crit }}$. It is found that

$$
\max _{\phi \leq \phi\left(\tau_{\text {res }, \text { crit }}\right)}\left(Q+U_{f s}\left(\phi, \phi_{\text {agg }, 0}\right)\right) \phi=\min _{\phi \geq \phi_{f}}\left(Q+U_{f s}\left(\phi, \phi_{\text {agg }}\left(\tau_{\text {res }, \text { crit }}\right)\right)\right) \phi
$$

where $\phi\left(\tau_{\text {res,crit }}\right)$ on the left is the $\phi$ value found by the minimisation on the right.

Once $\tau_{\text {res,crit }}$ and hence $\alpha_{\text {crit }}$ are determined (for any given $Q$ ), then if $\alpha \leq \alpha_{\text {crit }}$, one proceeds to find $\tau_{\text {res }}$ as normal via equations (16) and (22). On the other hand if $\alpha>\alpha_{\text {crit }}$, it becomes necessary to solve for

$$
\max _{\phi \leq \phi\left(\tau_{r e s}\right)}\left(Q+U_{f s}\left(\phi, \phi_{\text {agg }}\left(\tau_{\text {pre }}\right)\right)\right) \phi=\min _{\phi \geq \phi_{f}}\left(Q+U_{f s}\left(\phi, \phi_{\text {agg }}\left(\tau_{\text {res }}\right)\right)\right) \phi
$$

where $\phi\left(\tau_{\text {res }}\right)$ denotes the minimum found on the right hand side. This is to be solved alongside a constraint equation that the solids traverse a unit height between times $\tau_{\text {pre }}$ and $\tau_{\text {res }}$, which implies (in lieu of equation (16))

$$
1=\int_{\tau_{p r e}}^{\tau_{r e s}}\left(Q+U_{f s}\right) \mathrm{d} \tau
$$

To summarise the procedure for determining preshearing time is now as follows:

1. Determine whether the undensified maximum solids flux exceeds the fully densified minimum;

2. If not, find the critical densification rate such that the undensified maximum solids flux equals the corresponding densified minimum (at a critical residence time);

3. If the actual densification rate exceeds the critical rate, find the preshear time such that the presheared maximum solids flux equals the densified minimum correspond- 
ing to the actual residence time.

The above is clearly a fairly elaborate procedure for computing the preshear, and it is actually rather easier (and equivalent) just to vary $T_{\text {res }}$, and then to compute $T_{\text {pre }}$ and $l$ via (25)-(26), finally choosing $T_{\text {res }}$ so that the computed $l$ value equals the required target $\alpha$ (see also section 2.6.3). Remember also that preshearing can be avoided simply by reducing the suspension flux $Q$, and even at high fluxes, one can elect to forego preshear altogether (although the benefits of aggregate densification are not then realised, and there may be sacrifices in the underflow solids fraction that is achieved).

\section{Computational Methods}

The numerical techniques employed for solving the equations presented above are very simple and very standard ones (Press et al., 1992). For instance equations (19) and (22) can be solved by Newton-Raphson, whereas minimisations and maximisations (such as equations (17), (20), (21), (23), (25), (29), (30)) can be solved by applying NewtonRaphson to function derivatives. If (very occasionally) Newton-Raphson fails to converge to the correct root (owing to a poorly chosen initial guess) better initial guesses can be readily obtained by graphing the function in question. Integrals such as (12), (16), (26), (31) can be evaluated by quadrature.

\section{Results of Thickener Design Computations}

The results of thickener design computations are now presented, using the theories and methods described above. Section 4.1 analyses plots of solids flux, whilst section 4.2 analyses profiles of solids fraction. Finally section 4.3 considers the various thickener performance enhancements permitted by aggregate densification.

\subsection{Analysis of plots of solids flux}

In what follows the behaviour of solids flux vs solids fraction for three different suspension fluxes $(Q=0.025, Q=0.1$ and $Q=0.2)$ is analysed. As will be made apparent, these three distinct suspension fluxes are sufficient to highlight a variety of different types of possible system behaviour. 


\subsubsection{Solids flux when $Q=0.025$}

Figure 1(a) shows a plot of dimensionless solids flux $Q_{s} \equiv\left(Q+U_{f s}\right) \phi$ vs solids fraction $\phi$ for a suspension flux $Q=0.025$ at various different instants of time $T$ up to a total residence time $T_{\text {res }}=4$. This total residence time has been chosen large enough that material at the bottom of the settling zone is nearly fully densified. Based on the 'typical' parameter values presented in the appendix, these data correspond to a dimensional residence time of around $2000 \mathrm{~s}$ and a suspension flux around $2.5 \times 10^{-4} \mathrm{~m} \mathrm{~s}^{-1}$.

The operating solids fraction at the bottom of the settling zone corresponds to a local minimum of the $Q_{s}$ vs $\phi$ curve when $T=T_{\text {res }}$ (in this case $\phi=0.298$ with $Q_{s}=$ 0.00890). The underflow solids fraction $\phi_{u}$ can be obtained geometrically in the figure as the intersection between a horizontal line at position $Q_{s}$ and the line with slope $Q$. It turns out that $\phi_{u}=Q_{s} / Q=0.356$.

At any instant of time $T$ with $0 \leq T \leq T_{\text {res }}$, the solids fraction in the hindered settling zone can be read off from the graph, where it intersects the horizontal line at position $Q_{s}$. In order to maintain a dynamically stable configuration with less dense material above more dense material, one must choose the solution branch for which $\phi$ increases moving downwards through the hindered settling zone: the range of solids fractions specifically for the case $Q=0.025$ is indicated by the solid horizontal line in Figure 1(a).

\subsubsection{Solids flux when $Q=0.1$}

Figure 1(b) is similar to the case discussed above, with the same residence time, but a much larger dimensionless suspension flux value, i.e. $Q=0.1$ (based on the 'typical' parameter values in the appendix, the dimensional suspension flux would be $10^{-3} \mathrm{~m} \mathrm{~s}^{-1}$ ). The operating solids fraction at the bottom of the settling zone (again read off as the minimum of the $Q$ vs $\phi$ curve for the case $T=T_{\text {res }}$ ) is now smaller than before $\phi=0.194$, as is the underflow solids fraction $\phi_{u}=0.267$, but the operating solids flux is larger $\left(Q_{s}=0.0267\right)$.

Now consider the other curves shown on the figure (i.e. those corresponding to the undensified case and those for values of $T$ less than $\left.T_{r e s}\right)$. Note that the chosen suspension flux $Q=0.1$ now corresponds to a comparatively 'large' value, in the sense that for a 
relatively small further increase (up to a critical value $Q_{\text {crit }, 0}=0.148$ ) the undensified system would lose the local minimum (and maximum) in the $Q_{s}$ vs $\phi$ curve altogether. Instead it would have an inflection point (at $\phi=0.0870$, the inflection point of the original free settling flux curve), with $Q_{s}=0.0246$ at that point.

For $Q=0.1$ then, the undensified system has a (local) minimum and a (local) maximum, but these are fairly shallow. Specifically if the process were run without any aggregate densification whatsoever, the solids flux (corresponding to the shallow minimum) turns out to be $Q_{s}=0.0190$ and the underflow solids fraction would become $\phi=0.190$. The local minimum for the densified case gives better performance $\left(Q_{s}=0.0267\right.$ and $\phi_{u}=0.267$ as mentioned above). Nevertheless given the shallowness of the minima and maxima here, $\max _{\phi \leq \phi\left(T_{r e s}\right)}\left(Q+U_{f s}\left(\phi, \phi_{\text {agg, } 0}\right)\right) \phi$ (the local maximum of the undensified solids flux) is actually less than the target solids flux $Q_{s}$ (for the densified system).

This is precisely the scenario envisaged in equations (24) and (28) for which preshear becomes relevant. It turns out that in order to exploit aggregate densification the required preshear time in this case (with $Q=0.1$ and $T_{\text {res }}=4.0$ ) is $T_{p r e}=0.872$. Hence by time $T=1$ plotted in Figure 1(b), it is already possible to find a $\phi$ value satisfying equation (22), and the range of $\phi$ values covered for $T$ in the range $T_{\text {pre }} \leq T \leq T_{\text {res }}$ is indicated by the solid horizontal line in the figure.

Note that preshear only needs to be considered for comparatively large $Q$ values such as $Q=0.1$ here (it is never needed when $Q=0.025$ for instance). Notice moreover that, even for a large $Q$ value, preshear is not needed when $T_{\text {res }}$ is sufficiently small: the preshear time as a function of $T_{\text {res }}$ is plotted in Figure 2. Given that it was mentioned earlier (section 2.10) that preshearing may involve some engineering challenges, it is important to note in Figure 2 with $Q=0.1$, preshearing is avoided for $T_{\text {res }}$ less than about 0.4 .

\subsubsection{Solids flux when $Q=0.2$}

It is possible to increase $Q$ still further above and beyond the value in Figure 1(b). This is achieved in Figure 3 for a value $Q=0.2$ (based on the 'typical' parameter values in the appendix, the dimensional suspension flux would be $2 \times 10^{-3} \mathrm{~m} \mathrm{~s}^{-1}$ ). This $Q$ value can be considered to be 'very large' in the sense that, not only has the undensified flux 
curve lost its local minimum and maximum, but moreover the densified curves have also lost their local minima and maxima.

Recall that the solids flux delivered by the thickener is given by a minimum on the flux curve (see equation (17)), the minimum being sought in the domain $\phi \geq \phi_{f}$ (with $\phi_{f}$ being the feed solids fraction). However, once the (local) minimum on the flux curve is lost, the solution of equation (17) invariably occurs for $\phi=\phi_{f}$. The solids flux is said to be (Dickinson and Galvin, 2013) no longer constrained by the shape of the flux curve for $\phi>\phi_{f}$, but instead depends only on the conditions at the feed $\phi_{f}$. For the sake of illustration Figure 3 takes arbitrarily $\phi_{f}=0.05$, and the corresponding $Q_{s}$ (for a densified system with $\left.T_{\text {res }}=4\right)$ turns out to be 0.0343 . The underflow solids fraction $\phi_{u}$ becomes $Q_{s} / Q$ equal to 0.171 here.

It was discussed above with reference to Figure 1(a)-(b) how one could determine the range of solids fractions encountered in the settling zone of a thickener in the presence of aggregate densification. Specifically, given a $T_{r e s}$ value and a corresponding $Q_{s}$, one needed to consider $T$ values less than $T_{\text {res }}$, looking for $\phi$ values (at each $T$ ) that delivered the same $Q_{s}$. An immediate problem can be seen by applying this same procedure to Figure 3 however: the solids fraction $\phi$ would increase as $T$ decreases. This corresponds to a dynamically unstable situation for which more dense material is situated above less dense material. It is not clear what would happen to the suspension under these circumstances. The bed would presumably develop convective rolls which may enhance solids transport and water removal. On the other hand, the rolls could promote mixing of low and high solids fraction material which may be undesirable. Modelling these complex processes would require a 2-D and/or 3-D formulation of suspension mechanics, more sophisticated than the 1-D models considered here: this is beyond the scope of the present work.

For the $T_{\text {res }}=4$ system considered here, the only way one could access a $Q_{s}$ value as large as 0.0343 but avoid the above mentioned dynamically unstable configuration, is to suppose that all the aggregate densification takes place in a preshear unit, with no further aggregate densification taking place in the thickener itself. In other words $T_{\text {pre }}=T_{\text {res }}=4$. In engineering terms the task is no longer one of exploiting shear to improve operation of 
an existing thickener, but rather designing a completely new pretreatment or preshear unit for the feed. This is considered to be outside the scope of this study: the main focus here will be maintained on systems for which $Q$ is small enough that the $Q_{s}$ vs $\phi$ curve permits a local minimum (i.e. where the solids flux that is achieved is constrained (Dickinson and Galvin, 2013) by the flux curve, and at least some aggregate densification is permitted to take place in the thickener itself).

There is of course no physical difficulty in principle with using a suspension flux as high as $Q=0.2$, provided one operates in an undensified state and does not try to access solids fluxes as high as the value 0.0343 mentioned above. Figure 3 shows a case of undensified operation with $\phi_{f}=0.05, Q_{s}=0.0261$ and $\phi_{u}=Q_{s} / Q=0.130$. This is an admissible operating point for such a thickener, but (as a result of the high suspension flux $Q$ ), the underflow solids fraction $\phi_{u}$ really is quite modest, and moreover no benefits of aggregate densification whatsoever have been realised. Again such a regime is considered to be less relevant to this work, where the focus will be instead on smaller $Q$ values for which it is feasible to exploit aggregate densification within the thickener to enhance performance.

\subsection{Analysis of profiles of solids fraction}

Profiles of solids fraction $\phi$ vs position $Z$ (measured upwards from the bottom of the settling zone), respectively for $Q=0.025$ and $Q=0.1$, are shown in Figure 4(a)-(b). It must be emphasised that conventional Kynch theory predicts a fixed solids fraction in the settling zone. Profiles of varying $\phi$ vs $Z$ cannot be computed via conventional Kynch theory, but can be predicted by the densified Kynch theory considered here. Notice moreover that, even with densified Kynch theory, no corresponding profile could be computed in the case of a much larger $Q$ such as $Q=0.2$, because (as discussed in section 4.1.3) such a system has an operating solids fraction equal to the feed solids fraction. Hence only the profiles for $Q=0.025$ and $Q=0.1$ are considered here.

For each $Q$, various values of solids residence time are considered $T_{\text {res }}=\frac{1}{2}, 1,2$ and 4 . It is clear that increasing the solids residence time not only gives a larger solids fraction at the bottom of the settling zone but also increases the (dimensionless) height $l$ of that zone, a fact which is corroborated by the summary data in Table 1. Note that (based on the 
'typical' parameter values reported in the appendix) one unit of dimensionless distance corresponds to around $5 \mathrm{~m}$ dimensional distance, so the $l$ values in Table 1 correspond to a range of settling zone heights between $0.09 \mathrm{~m}$ and $3 \mathrm{~m}$. The cases $Q=0.025$ and $Q=0.1$ are now analysed in detail.

\subsubsection{Settling zone height for $Q=0.025$}

Each doubling of $T_{\text {res }}$ leads (according to Table 1 ) to roughly double the $l$ value: this is what one would expect from equation (12) since the integration range has been doubled. Nevertheless, in the case $Q=0.025$, doubling $T_{\text {res }}$ at smaller values of $T_{\text {res }}$ leads to slightly more than double the settling zone height, as the integrand $U_{f s}$ in equation (12) tends to increase with aggregate densification. On the other hand, doubling $T_{\text {res }}$ at larger values of $T_{\text {res }}$ leads to slightly less than double the zone height, because one starts to approach at the bottom of the settling zone (see Figure 4(a)) comparatively large solids fractions in the neighbourhood of $\phi=0.30$ for which settling speed $U_{f s}$ becomes quite small (observe that $U_{f s}$ is a sharply decreasing function of $\phi$ ).

\subsubsection{Solids fraction profiles in the settling zone for $Q=0.025$}

In addition to the settling zone total heights, the detailed shapes of the curves of solids fraction vs location in the settling zone (within Figure 4) are also of interest.

In all cases when $Q=0.025$ in Figure 4(a), the most rapid spatial changes in the solids fraction within the settling zone are seen towards the bottom of the settling zone: this is a manifestation of finding the operating flux at the bottom of the settling zone via a local minimum of the $Q_{s}$ vs $\phi$ curve.

The shape of the $T_{\text {res }}=4$ curve is qualitatively different from the other curves at smaller $T_{\text {res }}$. In particular the $T_{\text {res }}=4$ curve displays an inflection point at which the derivative $\mathrm{d} \phi / \mathrm{d} Z$ is a minimum. In engineering terms, this means that the settling zone is gaining significant height without the benefit of much increase in the value of solids fraction. According to equations (8)-(9), in the limit of large $T$, the value of $\phi_{\text {agg }}$ changes comparatively little with $T$, so the implication from equation (19) is that $\phi(T)$ likewise changes comparatively little with $T$ (or equivalently with $Z$ ). This is what produces the 
above mentioned inflection.

It was mentioned earlier that the $T_{\text {res }}=4$ case in Figure $4(\mathrm{a})$ reaches a solids fraction of nearly 0.30 at the bottom of the settling zone. At solids fractions this large however, it is possible to query whether in a real suspension it is still valid to treat the suspension as being Kynchian (as has been done here), or whether additional network stresses (which are ignored here) might need to be taken into account (Buscall and White, 1987; Landman and White, 1994). The study of Lester et al. (2005) for instance assumed an (undensified) gel point at solids fraction 0.22 . As the flocs densify, the gel point scales proportional to $D_{a g g}^{-3}$. With the $D_{a g g, \infty}$ value assumed here (namely 0.9), the gel point of the corresponding fully densified system would be at a solids fraction of around 0.30. As noted earlier (see section 2.3), this is material property dependent (Usher et al., 2013) and whereas some suspensions reach a gel point for solids volume fractions even less than 0.1 , other coarser suspensions do not reach a gel point except for solids fractions in excess of 0.3 . The values of the gel point utilised here are thought to be typical of a range of mineral suspensions (Gladman et al., 2006).

In the case studied here, the material at the bottom of the settling zone as indicated in Figure 4(a) would be just barely approaching the point of forming an exceedingly weak gel. Material higher up in the settling zone is certainly not gelled though: in Figure 4(a) in the case $T_{r e s}=4.0$, undensified material at the top of the settling zone is predicted to have a solids fraction around 0.13 , which is much less than the undensified gel point (solids fraction 0.22). For comparison, the operating point of the corresponding undensified thickener (still with $Q=0.025$ ), which is indicated by a vertical line in Figure 4(a), itself obtained via the local minimum of the corresponding undensified curve in Figure 1(a), is actually a solids fraction of 0.21 , which is likewise just less than the undensified gel point (solids fraction 0.22 ).

It seems reasonable then to ignore gelation for these data, but one certainly could not set the suspension flux $Q$ much lower than the current value $Q=0.025$ without explicitly considering gelation, because lowering $Q$ tends to increase solids fractions. Interestingly Figure 4 shows that the range of solids fractions encountered in the settling zone of the 
densified thickener straddles the operating point of the undensified one (i.e. $\phi \sim 0.21$ as mentioned above): compared to the undensified case, densified system material is predicted to be less concentrated higher up but more concentrated lower down. What matters fundamentally to thickener performance however is the state at the bottom of the settling zone, and the underflow solids fraction that this implies: based on that criterion, aggregate densification can be shown to enhance performance (as will be considered in detail later in section 4.3).

\subsubsection{Jumps in solids fraction for $Q=0.025$}

Note that each case plotted in Figure 4(a) also shows a jump in solids fraction that is permitted at the top of the settling zone. Such a jump comes about because the system (and in particular the still undensified state at the top of the settling zone) allows multiple possible values of $\phi$ for a given $Q_{s}$. This is evident for instance in Figure 1(a) (the specific case with total residence time $T_{\text {res }}=4$ ). The solids fraction at the top of the settling zone corresponds to a value around 0.13 (the left hand end of the solid horizontal segment in Figure 1(a) that indicates the range of solids fractions permitted in the settling zone), but the same flux can also be delivered with much smaller solids fraction of around $\phi \sim 0.01$. One could in fact dilute the feed down to this solids fraction without affecting the thickener solids throughput. The suspension would then be dilute between the top of the solids fraction profile and some arbitrary feed point higher up. Because these dilute states have such low solids fraction (around $\phi \sim 0.01$ as indicated in Figure 4(a)), the solids move through the dilute zone very quickly. As a result, the solid aggregates incur little densification whilst moving through the dilute zone, and it is justified to suppose that the top of the solids fraction profile corresponds to a still undensified state (as has been assumed here). The solids move much more slowly through and spend much more time in the hindered settling zone, which is located underneath the dilute zone: therefore much more densification is able to take place in the hindered settling zone than would ever occur in the dilute zone.

In a similar fashion to what has been discussed above, the $Q_{s}$ vs $\phi$ curve for a system with no aggregate densification whatsoever (also indicated in Figure 1(a)) likewise permits 
multiple solutions for $\phi$ at given $Q_{s}$, and so permits a corresponding jump in solids fraction from a dilute to a less dilute state, and this jump is also indicated in Figure 4(a) from around $\phi \sim 0.01$ to $\phi \sim 0.21$. The jump is actually larger in the undensified case than in the densified ones, but, in the densified cases, the solids fraction continues to increase moving downwards through the settling zone, so at the bottom of the settling zone, the solids fractions have reached higher levels for the densified systems.

\subsubsection{Analysis of the settling zone for $Q=0.1$}

Now turn to the case $Q=0.1$ as presented in Figure 4(b). Operating without aggregate densification is of course possible here, and again a jump in solids fraction is admitted (the jump being indicated in Figure 4(b) from $\phi \sim 0.032$ to $\phi \sim 0.135)$. As before however, operational benefits are expected if aggregate densification occurs, and the solids fractions at the bottom of the settling zone is seen to increase with $T_{\text {res }}$. The solids fractions accessed all now appear to be well below the gel point. Notice however that the solids fraction at the top of the settling zone now also increases with $T_{\text {res }}$, which is a contrast from the case in Figure 4(a). The difference is due to the fact that exploiting aggregate densification in the $Q=0.1$ system in Figure 4(b) involves significant preshear which permits increases in solids fraction even at the top of the hindered settling zone. Data on preshearing times are presented in Table 1. Rapid spatial changes in solids fraction are now seen both at the bottom and top of the settling zone: this is a manifestation of having a local minimum of $Q_{s}$ vs $\phi$ at time $T_{\text {res }}$ and a local maximum at time $T_{\text {pre }}$. A further consequence of the $Q_{s}$ vs $\phi$ curve achieving a local maximum at time $T_{\text {pre }}$ is that, at that particular time, there is not any more dilute state that is able to deliver an equivalent solids flux of presheared material. Hence in Figure 4(b), unlike Figure 4(a), for residence times $T_{\text {res }}=\frac{1}{2}, 1,2$ or 4 , no jump in solids fraction is indicated between a more dilute system and the top of the settling zone. These jumps do however reappear if the residence time $T_{r e s}$ is reduced sufficiently to eliminate preshear altogether: in particular they occur (as mentioned above) for $T_{\text {res }} \rightarrow 0$ which has no preshear (although the $T_{\text {res }} \rightarrow 0$ system also has no aggregate densification, and hence derives no benefit from the aggregate densification process). 
It is also clear from Table 1 that with each doubling of $T_{\text {res }}$, the settling zone height roughly doubles. For small $T_{\text {res }}$ however, doubling $T_{\text {res }}$ now leads (in the case $Q=0.1$ ) to slightly less than double the zone height. The reason is that in the presence of preshear, zone height is computed via equation (26) rather than equation (12). The duration spent in the settling zone $T_{\text {res }}-T_{\text {pre }}$ may not quite double even though $T_{\text {res }}$ does. At arbitrarily large $T_{\text {res }}$ however, $T_{\text {pre }}$ saturates, at a value given by solving equation (12) but with $\phi_{\text {agg }, \infty}$ replacing $\phi_{\text {agg }}\left(T_{\text {res }}\right)$ on the right hand side. Doubling $T_{\text {res }}$ then more than doubles the duration in the settling zone $T_{r e s}-T_{\text {pre }}$, and it is evident from Table 1 that (for larger $\left.T_{\text {res }}\right)$ doubling $T_{\text {res }}$ slightly more than doubles zone height $l$.

\subsubsection{Generalisation to a conical-shaped thickener}

Figure 1 and Figure 4 have both been obtained for the case of a suspension in a straight-sided thickener, where $Q$ and $Q_{s}$ are spatially uniform. Here, for completeness, it is explained how one could generalise this to the case of a conical shaped-thickener (although, for the sake of brevity, no results for that case will be presented). In a conical thickener, $Q$ and $Q_{s}$ would vary inversely with the thickener cross-section. Equation (11) relates the position in the thickener $Z$ to the time in the settling zone $T$, and hence, for a conical thickener, relates the position-dependent $Q$ and $Q_{s}$ to time $T$. One could generate settling flux curves analogous to those in Figure 1 albeit now with different $Q$ for each $T$, and read off the $\phi$ values that deliver the required $Q_{s}$ (which now also varies with $T$ ): thus $\phi$ vs $T$ and hence $\phi$ vs $Z$ profiles could be generated analogous to Figure 4 . The main difficulty here is that $Q$ and $Q_{s}$ are most readily expressed in terms of $Z$, whereas $U_{f s}$ (which appears in equation (11)) involves $\phi_{\text {agg }}$, which is most readily expressed in terms of $T$. For the straight-sided thickener, the right hand side of equation (11) could be determined at any $T$ without knowing $Z$ in advance. For the conical thickener however, that right hand side can only be evaluated as the equation itself for $Z$ vs $T$ is solved.

\subsection{Performance enhancements due to aggregate densification}

Densifying a suspension via raking should enhance thickening performance. There are however various different ways of quantifying this performance enhancement as is 
explained in the sections to follow.

\subsubsection{Varying shear residence time $T_{\text {res }}$ at fixed suspension flux $Q$}

One way of representing the performance enhancement due to aggregate densification is shown in Figure 5. Specifically for the suspension fluxes $Q=0.025$ and $Q=0.1$ considered above, the figure plots the underflow solids fraction $\phi_{u}$ vs $T_{r e s}$. In each case $\phi_{u}$ in the large $T_{\text {res }}$ limit is about $40 \%$ higher than for the undensified case. This $40 \%$ enhancement is of course particular to the floc diameter ratio $D_{a g g, \infty}=0.9$ considered here: smaller values of $D_{a g g, \infty}$ would permit even greater enhancements.

\subsubsection{Varying shear residence time $T_{r e s}$ at fixed underflow solids fraction}

Alternatively one can compute the performance enhancements not at fixed $Q$, but rather, using equation (20), at fixed underflow solids fraction $\phi_{u}$. This is particularly relevant since many thickeners operate to a fixed rake torque. At underflow concentrations well above from the gel point, this is often a fixed solids fraction for any one material that has a given level of flocculation. The yield stress of such suspensions shows a strong correlation to rake torque (Rudman et al., 2008). At underflow concentrations close to the gel point, the behaviour is more subtle, especially in light of the changes to the gel point as a result of aggregate densification. Data are presented in Figure 6 for values $\phi_{u}=0.25$ and $\phi_{u}=0.35$ (although note a former reservation - see section 4.2.2 - regarding whether it is always appropriate to ignore suspension network stresses in the latter case, as a gel may be on the point of forming in the settling zone).

For $\phi_{u}=0.25$, the (nearly) fully densified case at $T_{r e s}=4$ has a $Q_{s}$ roughly 4 times that of the undensified case. For $\phi_{u}=0.35$ the relative increase in $Q_{s}$ (undensified to fully densified) is 13 times, although the undensified case now starts from an exceedingly small solids flux (see Figure 6). The result is that the fully densified $Q_{s}$ for $\phi_{u}=0.35$ is, in order of magnitude terms, comparable with the undensified $Q_{s}$ for $\phi_{u}=0.25$, with only around $30 \%$ difference between them. Again these results are of course particular to

the value $D_{a g g, \infty}=0.9$ assumed here: even smaller $D_{a g g, \infty}$ would enhance yet more the densified performance. 


\subsubsection{Solids fraction $Q_{s}$ vs underflow solids fraction $\phi_{u}$ for various $T_{\text {res }}$}

Results of thickener models are often presented (Usher and Scales, 2005) via a graph of $Q_{s}$ vs $\phi_{u}$. In Figure 7 such a plot is shown.

Focus first on the undensified case: two branches of solutions are shown in Figure 7(a). One is a low solids flux and high underflow solids fraction branch, and corresponds to finding a local minimum of the solids flux vs solids fraction curve via the undensified analogue of equation (17). The other is a high solids flux and low underflow solids fraction branch, and corresponds to finding the minimum of equation (17) at the feed solids fraction $\phi_{f}$. Here $\phi_{f}$ has been taken arbitrarily to have the value 0.05 . The first branch of solutions is said to be constrained by the shape of the flux curve, and the second branch is said to be unconstrained (Dickinson and Galvin, 2013).

As solids flux is increased, the system follows initially the first branch, but then switches to the second branch at the point where the two branches intersect. It is clear from the shape of the curves, that when the second branch is attained, the system starts to sacrifice significantly the underflow solids flux that can be achieved. Delaying the switch to the second branch (if possible) is desirable. It turns out that the branch intersection point is actually sensitive to the value of $\phi_{f}$. For an appropriately chosen 'optimal' $\phi_{f}$ value, the furthest possible that one can move along the first branch is to the point where the solids flux vs solids fraction curve ceases to have a local minimum and maximum ${ }^{2}$, beyond which that particular branch ceases to exist, and the thickener is invariably operated at the feed solids fraction (rather than at a locally minimum flux).

The above discussion has concerned the undensified case. A similar picture arises for densified systems at different values of the shear residence time $T_{\text {res }}$ (undensified, $T_{\text {res }}=\frac{1}{2}, 1$ and 2 , as well as the fully densified case). As $T_{r e s}$ increases, the graphs are shifted upwards and to the right. This is indicative of a performance enhancement.

For each $T_{\text {res }}$ again there are two branches of solutions: a constrained and an unconstrained branch analogous to those discussed above. The unconstrained branches with

\footnotetext{
${ }^{2}$ Moving along the branch all the way to this end point requires that the feed solids fraction matches the inflection point on the original free settling flux curve. As mentioned in section 4.1.2, this is a solids fraction of 0.0870 in the undensified case.
} 
non-zero $T_{\text {res }}$ are of limited interest here, because they actually require all the aggregate densification to have occurred via preshear, with no further aggregate densification in the thickener itself. Otherwise these branches would imply dynamically unstable conditions, with more dense material above less dense material (see also sections 4.1 .1 and 4.1.3). If no aggregate densification takes place in the thickener itself (but instead only in an upstream preshear unit), one is no longer trying to improve the operation of a thickener per se, but instead is designing a pretreatment process for the feed: this falls beyond the scope of the present discussion, and may entail considerable engineering challenges. For present purposes, it is expected that whenever the unconstrained operation branch is accessed, that would mostly likely occur without the benefit of any aggregate densification.

The focus here therefore is on the constrained branch with various non-zero $T_{\text {res }}$. This is shown in detail in Figure 7(b). Note that most of the data presented (i.e. all of those below $Q_{s} \approx 0.02$ ) now do not involve preshear. The critical solids fluxes $Q_{s}$ for preshear are indicated in the figure and seem to be only very weakly sensitive to $T_{r e s}$ (except of course in the limit $T_{\text {res }} \rightarrow 0$, where preshear is never required, but where no aggregate densification occurs either, and hence no benefit of aggregate densification is ever realised).

For each $T_{r e s}$ the value of $Q_{s}$ corresponding to the point at which the $Q_{s}$ vs $\phi$ curve loses its local maximum and minimum is also indicated. As discussed above, this is the point at which the system ceases to be constrained by the flux curve (Dickinson and Galvin, 2013) even for an 'optimally' chosen feed solids fraction. Once this level of solids flux is attained all the aggregate densification occurring is achieved via the preshear, with no further aggregate densification taking place in the thickener itself (i.e. the preshear time equates to the total residence time in the shear field). Moreover, even though yet higher values of solids flux can in principle be accessed by switching the solution branch, this could possibly entail (again as discussed above) foregoing the benefits of aggregate densification altogether.

\subsubsection{Solids fraction $Q_{s}$ vs underflow solids fraction $\phi_{u}$ for various $\alpha$ or $l$}

Recall (from section 2.6) that there were two ways to formulate the densified thickener problem: in one formulation there was a fixed extent of aggregate densification (i.e. given 
$T_{\text {res }}$ ) but the dimensionless settling zone height was allowed to vary (i.e. variable $l$ ). In the second formulation a densification rate parameter $\alpha$ was fixed, but time in the settling zone was allowed to vary in order to meet a constraint on the settling zone height (equations (16) or (31)). This of course presupposes that a settling zone height is well defined by the model, which (out of the two solutions branches discussed in section 4.3.3) is only true for the so called constrained branch. Subject to this condition, remember however that both formulations were equivalent. Thus using the first formulation, one could vary $T_{\text {res }}$ and thereby vary $l$ until the computed value of $l$ equalled the target $\alpha$ value given in the second formulation.

A look up table of $Q_{s}$ vs $\phi_{u}$ has therefore been generated for a large number of possible $T_{\text {res }}$ values, and data have been interpolated between different $T_{\text {res }}$ values to generate performance curves at fixed $\alpha$. These are plotted on Figure 8 for a variety of $\alpha$ values between $\alpha=0.02$ and $\alpha=0.2$. Physically comparing different $\alpha$ corresponds to considering different rates of raking a suspension (for a suspension of a given height) or equivalently considering suspensions of differing heights (at a given rate of raking). For the 'typical' parameter values discussed in the appendix, the range of values $\alpha=0.02$ through $\alpha=0.2$ would correspond to settling zone heights between $0.1 \mathrm{~m}$ and $1 \mathrm{~m}$.

In Figure 8, focus first on $Q_{s}$ values below $Q_{s} \approx 0.02$ for which it is known that the system benefits from aggregate densification even without preshear. It is clear that in this regime increasing $\phi_{u}$ or equivalently decreasing $Q$ leads to data migrating from the neighbourhood of the undensified curve $T_{\text {res }} \rightarrow 0$ to the neighbourhood of the fully densified one $T_{\text {res }} \rightarrow \infty$.

This can also be seen from equation (16). Increasing $\phi_{u}$ (and hence increasing $\phi$ ) leads to decreases in $U_{f s}$, whilst decreasing $Q_{s}$ (which accompanies increases in $\phi_{u}$ ) correlates with decreasing $Q$. Hence the integrand of equation (16) decreases as $\phi_{u}$ increases, so the integration range must grow to compensate: $\tau_{\text {res }}$ and $T_{\text {res }} \equiv \alpha \tau_{\text {res }}$ both grow, hence the observed migration towards the fully densified case. Physically this merely demonstrates that systems with lower solids velocity require more time to cover a given distance.

Now consider $Q_{s}$ values above $Q_{s} \approx 0.02$. Preshear can now be utilised to access solids 
fluxes higher than the maximum value 'accessible' to the undensified system ${ }^{3}$ (value quoted previously in section 4.1 .2 as $\left.Q_{s}=0.0246\right)$. Accessing this higher solids flux regime is an operational option for thickeners operating with an underflow solids at or about the gel point and although classical Kynchian theory would disallow such a move, the aggregate densification approach shows why many operational thickeners indeed derive a benefit.

The more that $Q_{s}$ is increased above the above mentioned level, the more preshear should occur ${ }^{4}$. However since total residence time $\tau_{\text {res }}$ must be at least as high as preshear time $\tau_{\text {pre }}$, it is clear that increasing $Q_{s}$ now requires $\tau_{r e s}$ to increase. As $Q_{s}$ increases towards the maximum permitted solids flux for a fully densified state on the solution branch in question (which can be shown to be $Q_{s}=0.0375$ with a corresponding underflow solids fraction $\phi_{u}=0.228$ ) all the curves on Figure 8 with different values of $\alpha$ converge on the same point. In this maximum permitted $Q_{s}$ limiting case, the constraint equation (31) now applies, but the integrand of that equation approaches a constant fully densified value, with the result that $\tau_{\text {res }}-\tau_{\text {pre }}$ is likewise constant. It is convenient at this point to change variables. Recognising that $T_{\text {res }}=\alpha \tau_{\text {res }}$ and $T_{\text {pre }}=\alpha \tau_{\text {pre }}$ and $\alpha=l$, it is possible to deduce that the time spent in the hindered settling zone $T_{r e s}-T_{\text {pre }}$ is directly proportional to zone height $l$, but increases in $l$ have no bearing on the system performance (i.e. no bearing on $Q_{s}$ or $\phi_{u}$ ). In other words if the system is presheared to near full densification, there is little point in having a tall thickener (at least in this ungelled system where network stresses are ignored).

\section{Discussion}

This work has performed a modelling study of the thickening behaviour of an ungelled 'Kynchian' suspension (i.e. one without compressive network forces), a model that would

\footnotetext{
${ }^{3}$ Specifically this maximum 'accessible' value refers to the highest solids flux that would be permitted for operation that is constrained (Dickinson and Galvin, 2013) by the shape of the flux curve (see also sections 4.1.3 and 4.3.3). Such operation gives robust control of the solids flux delivered in case of any fluctuations in solids fraction (see section 2.7).

${ }^{4}$ Note that the formal limit $\alpha \rightarrow 0$ corresponds to no densification when $Q_{s}<0.0246$, but for $Q_{s}>0.0246$ corresponds instead to preshear time equal to total residence time in the shear field, as is obvious from considering the integration limits in equation (26) remembering the equivalence between the parameter $l$ in equation (26) and the parameter $\alpha$.
} 
be permissible only for suspensions with comparatively low solids fractions. The model has treated steady state thickening (in the Eulerian sense), but has included the effect of flocs within the suspension being subjected to the shearing action of rakes and, as a result, becoming densified over time (in the Lagrangian sense). The model has been applied to study the behaviour of a straight-sided thickener, although can be generalised to a conical-shaped thickener without too much additional difficulty.

A model for floc densification previously employed in the literature (Usher et al., 2009; van Deventer et al., 2011; Zhang et al., 2013a,b) has been used. Surprisingly (given the fact that the model in question tends to predict significant benefits of floc densification for comparatively low solids fractions) it has typically previously been used to study thickening of higher solids fraction gelled/networked suspensions, not lower solids fraction Kynchian ones as are considered here.

The aggregate densification model employed here provides more information than standard (undensified) theories for thickening of Kynchian suspensions: in particular it predicts settling zone heights and profiles of varying solids fraction vs position in the settling zone. These heights and profiles are most readily obtained by specifying the state of the suspension at the bottom of the settling zone (at which the greatest amount of aggregate densification has taken place) and then computing the various suspension states above this, maintaining a spatially uniform solids flux.

Significant performance enhancements of thickeners due to aggregate densification are predicted, for instance a $40 \%$ increase in underflow solids volume fraction (for a given suspension throughput) or increases in solids fluxes by a factor between 4 and 13 (for a given underflow solids volume fraction). Such performance improvements however come at the cost of an increase in the settling zone height, as residence times in the settling zone must be longer. These performance enhancements are also sensitive to the densification parameters employed, in particular to the ratio between the diameters of fully densified flocs and undensified flocs (the fully densified state being defined here as the point at which the floc diameter ceases to change with time). As this diameter ratio decreases, the predicted performance enhancement would increase still further. 
Moreover solids fluxes 'unattainable' by undensified systems ${ }^{5}$ can be attained using densified ones, although these may involve some preshearing upstream of the thickener feed, with the total residence time spent in the shear field being measured as the sum of the preshearing time and the time in the settling zone. It is even possible to contemplate a very high flux situation which can still benefit from aggregate densification, provided the aggregate densification taking place during preshear totally dominates any subsequent aggregate densification occurring in the thickener itself: this however is considered to be outside the scope of the present study as it involves exclusively pretreatment options for the thickener feed, rather than improvement of thickener performance per se.

If one is willing to forego any operational benefits from aggregate densification, then it is possible to operate a thickener at any solids flux without preshear. If one wants to exploit aggregate densification however, the requirement or otherwise for preshearing is determined primarily by the target solids flux, with just a very weak dependence on the residence time in the shear field itself. Indeed preshear can always be avoided if the solids flux is set sufficiently low. Given that the requirement for even modest amounts of preshear may present engineering challenges (particularly if it involves retrofitting onto an existing thickener design) identifying the parameter domain for which preshear can be avoided is useful.

Regardless of whether the system is (or is not) presheared, performance curves can be plotted for solids flux $Q_{s}$ vs underflow solids fraction $\phi_{u}$. These curves can be parameterised by the extent of aggregate densification (or analogously by the residence time for which the system is subject to densification), varying from the undensified state through to the fully densified one.

It is more straightforward however to represent the curves using a densification rate parameter (for a fixed settling zone height) or a settling zone height parameter (for a given densification rate). When cast in dimensionless form, these two parameters become the

\footnotetext{
'Unattainable' here should be taken to mean unattainable to systems that are operated in a state that is constrained by the shape of the flux curve for solids fractions in excess of the feed solids fraction. Accessing higher solids fluxes remains possible but involves, not only sacrifices in underflow solids fraction, but also loss of robustness of the solids flux delivered in the event of any fluctuations in solids fraction.
} 
same. For a fixed value of this (dimensionless) rate parameter, as the target solids fraction $\phi_{u}$ grows (and hence the solids flux $Q_{s}$ falls), the solids velocities also fall, and hence the time required to cover a given settling height must grow. The extent of aggregate densification (which is determined by the product between the aforementioned dimensionless rate parameter and the dimensionless settling time) then grows: the system migrates from a nearly undensified state to a nearly fully densified one. This migration occurs more readily for taller settling zones, and/or for systems that are raked/sheared more rapidly, although the raking must not be so rapid as to tear aggregates apart altogether.

However towards the upper end of the flux regime of interest the system makes the same transition as $Q_{s}$ grows. This is the result of increasing amounts of preshear being imposed in order to exploit the benefits of aggregate densification. Moreover in the limit of sufficiently large fluxes, the thickener performance becomes independent of the densification rate parameter (or equivalently the settling zone height parameter) since the suspension then must be presheared almost to full densification even before entering the thickener, or in another way of thinking, the benefits of aggregate densification cannot be realised in a conventional thickener (without some significant redesign to achieve the necessary preshear).

It is important to recall the restriction that the 'Kynchian' model considered here only treats the case of suspensions at low enough solids fraction such that they do not form a weight bearing gel, otherwise more complex theories of thickening with aggregate densification are required, as have been studied elsewhere (Usher et al., 2009; Zhang et al., 2013a,b). Nonetheless, in some of the computations presented here, particularly those where solids fluxes are far below the level at which preshear would ever be imposed, solids fraction towards the bottom of the settling zone in a densified thickener, just barely approaches levels comparable with the densified gel point. Any further reductions in solids flux (which tend to be accompanied by increases in solids fraction) would certainly demand theories that account for gelled suspensions. The presence of a gelled network which tends to resist compression will likely cause the dewatering performance achieved to be less good than what has been predicted here for an unnetworked material in the 
absence of any compressional hindrance.

\section{Conclusions}

This work has discussed how the mathematical description of suspensions at low solids fraction differs from that of suspensions at higher solids fraction: at higher solids fraction suspensions tend to form a gel which can support compressive stresses (Buscall and White, 1987). Lower solids fraction suspensions are not gelled, and do not exhibit compressive stresses. Instead their rheology is normally modelled very simply via so called Kynch theory (Kynch, 1952) which employs a relation that describes how much flocs or aggregates in the suspension hinder the settling of neighbouring flocs. The more that flocs hinder the settling of their neighbours, the more difficult a given suspension becomes to dewater.

Kynch theory for ungelled suspensions (and extensions thereof that incorporate compressive stress terms for higher solids fraction gelled suspensions) can be used for engineering design of dewatering equipment. One of the simplest types of equipment to design is a so called continuous thickener, in which suspension is fed into the device, with clear water being drawn off the top, and thickened material (with a solids fraction greater than that of the feed) being drawn off the bottom. Indeed the operation of a thickener (Talmage and Fitch, 1955) is very easy to predict in the context of Kynch theory (and/or generalisations thereof that incorporate compressive stresses if required (Usher and Scales, 2005)). One specifies a suspension flux solids through the thickener, and the theory predicts the solids flux achieved and the underflow solids fraction at the bottom. Invariably a trade off is found between the solids flux and the underflow solids fraction: a high suspension flux permits a high solids flux but leads to a comparatively modest underflow solids fraction, whereas a lower suspension flux gives a lower solids flux but a higher solids fraction out the bottom.

Conventional Kynch theory however does not account for the fact that the microstructure of flocs is not necessarily fixed, but instead can evolve over time. In particular raking a suspension may induce changes in floc structure: specifically aggregates densify, i.e. become more compact, under the shearing action of rakes (Usher et al., 2009). Densification 
of individual aggregates opens up wider channels between aggregates, thereby facilitating the escape of water from the suspension. Thus the state of a suspension and its dewaterability depend not only upon the solids fraction, but also upon the extent of aggregate densification. This tends to produce a very significant effect: even a modest increase in the width of channels between aggregates can enhance dewatering rates dramatically (Usher et al., 2009).

Relations are available between the extent of aggregate densification and residence time in the shear field within a raked suspension (Usher et al., 2009; van Deventer et al., 2011). Hence dewaterability becomes a function of solids fraction and residence time in the shear field. The aim of the present work therefore has been to redo thickener design calculations using a modified Kynch theory that accounts explicitly for residence time in a raked thickener.

The most important finding is that dramatic increases in thickener performance are possible. The performance enhancement is most simply expressed via a graph of the solids flux vs the solids fraction in the underflow of the thickener: as residence time increases, the graph is pushed towards higher solids fluxes and higher solids fractions.

The next most important finding is that the modified Kynch theory presented here predicts a profile of solids fraction vs position in the thickener, and hence makes predictions for the thickener height required to attain a prescribed underflow solids fraction. This is a surprising outcome because conventional Kynch theory in the absence of aggregate densification makes no such predictions: it determines an operating solids fraction within the thickener, but not a solids fraction profile, and does not specify any thickener height requirement.

Interestingly generalisations of Kynch theory taking account of compressive effects in gelled suspensions (but without necessarily invoking aggregate densification) do predict solids fraction profiles and thickener height requirements (Usher and Scales, 2005). Those predictions are however associated with the gradients of solids fraction needed to achieve the gradients of compressive stress that produce an overall force balance. The mechanism for predicting a solids fraction profile and hence thickener height requirement is however 
rather different in the case that has been studied here of a Kynchian i.e. ungelled suspension subject to aggregate densification. In that case, different points in the thickener have different residence times (and hence different extents of aggregate densification). This in turn implies that a given solids flux through the thickener is actually achieved with a profile of solids fraction which is not uniform across the settling zone.

\section{Acknowledgements}

The authors would like to acknowledge infrastructure support through the University of Melbourne and the Particulate Fluids Processing Centre, a Special Research Centre of the Australian Research Council along with financial support and discussions through AMIRA project P266. Part of this work also was carried out whilst PG was a Royal Academy of Engineering/Leverhulme Trust Senior Research Fellow and funding from the fellowship is gratefully acknowledged.

\section{Appendix}

In section 2.6 various different characteristic velocity, length and time scales relevant to suspension thickening were considered. Here it is quantified what those scales imply for a 'typical' suspension.

A floc of assumed radius 100 microns, with an assumed average density $50 \%$ higher than that of surrounding water settles (in the absence of any other flocs) according to Stokes settling law (see e.g. Batchelor (1967)) at a speed $u_{\text {Stokes }}$ of roughly $10^{-2} \mathrm{~m} \mathrm{~s}^{-1}$, assuming the surrounding water has centipoise viscosity. This 'unhindered' speed $10^{-2} \mathrm{~m} \mathrm{~s}^{-1}$ is however probably an overestimate of the true settling speed in a suspension, given that at a solids fraction $\phi$ of around 0.2 , equations (1)-(2) (with the chosen exponent $N=20$ ) predict batch settling speeds around 100 times lower than the Stokes speed, and even at a solids fraction of around 0.1, batch settling speed is still 10 times slower than Stokes. At any given solids fraction, thickening of course proceeds faster than batch settling (owing to the addition of the suspension flux) but even for the case of a 'large' $Q$ (e.g. the undensified critical value $Q_{c r i t, 0}=0.148$ mentioned in section 4.1.2), it can be shown that 
the total solids speed is just barely double the batch settling speed (at the corresponding operating solids fraction).

Now turn to time and length scales. The work of van Deventer et al. (2011) considered values of the rate parameter $A$ up to $10^{-2} \mathrm{~s}^{-1}$, but finds a best fit to data with an $A$ value around $2 \times 10^{-3} \mathrm{~s}^{-1}$. The corresponding time scale $A^{-1}$ is $500 \mathrm{~s}$. The characteristic length scale $u_{\text {Stokes }} / A$ meanwhile is on the order of about $5 \mathrm{~m}$. It would however be rare to find a thickener as tall as $u_{\text {Stokes }} / A$ given (as mentioned above) that hindered settling speeds at solids fractions typically encountered in thickening are substantially lower than $u_{\text {Stokes }}$. In other words, the ratio $l$ between the settling zone height $L$ and the characteristic length scale $u_{\text {Stokes }} / A$ will normally be rather less than unity.

Recall from section 2.6.3 however that this ratio could be identified with the parameter $\alpha \equiv A L / u_{\text {Stokes }}$, as considered in Figure 8. The range of $\alpha$ values considered in Figure 8 is in fact $\alpha=0.02$ through $\alpha=0.2$. If $u_{\text {Stokes }} / A$ is roughly $5 \mathrm{~m}$ (as above), the data in this figure correspond to settling zone heights between $0.1 \mathrm{~m}$ and $1 \mathrm{~m}$.

\section{Nomenclature}

$A$ densification rate parameter

$D_{\text {agg }}$ diameter ratio between densified and undensified flocs; a function of time

$D_{a g g, \infty}$ diameter ratio between fully densified and undensified flocs (here $D_{a g g, \infty}=0.9$ )

$L$ settling zone height in thickener

$l$ dimensionless settling zone height

$N$ exponent in the hindered settling factor formula (here $N=20$ )

$q$ suspension flux

$Q$ dimensionless suspension flux

$Q_{s}$ dimensionless solids flux

$Q_{\text {crit }, 0}$ a critical suspension flux at which the undensified solids flux curve loses its local minimum and maximum

$r$ hindered settling factor

$r_{0}$ hindered settling factor in undensified system

$t$ time

$t_{\text {res }}$ residence time in the shear field 
$T$ dimensionless time

$T_{\text {res }}$ dimensionless residence time in the shear field (in the absence of any preshear, this is also the same as the time spent in the thickener settling zone)

$T_{\text {pre }}$ dimensionless preshear time

$u_{f s}$ floc 'free settling' speed; a function of solids fraction

$U_{f s}$ dimensionless floc free settling speed

$u_{\text {Stokes }}$ Stokes settling speed for an isolated floc

$z$ vertical coordinate

$Z$ dimensionless vertical coordinate

$\alpha$ dimensionless densification rate parameter

$\alpha_{\text {crit }}$ a critical densification rate that separates systems that involve preshear from those that do not; a function of suspension flux

$\zeta$ dimensionless vertical coordinate (in an alternate scaling)

$\tau$ dimensionless time (in an alternate scaling)

$\tau_{\text {res }}$ dimensionless residence time in the shear field (in an alternate scaling)

$\tau_{r e s, 0}$ dimensionless residence time that would apply to an undensified system

$\tau_{\text {res, } \infty}$ dimensionless residence time that would apply to a fully densified system

$\tau_{\text {res, crit }}$ a critical residence time that occurs when the densification rate is $\alpha_{\text {crit }}$; a function of suspension flux

$\tau_{\text {pre }}$ dimensionless preshear time (in an alternate scaling)

$\phi$ solids fraction

$\phi_{\text {agg }}$ solids fraction within aggregates; a function of time

$\phi_{\text {agg }, 0}$ solids fraction within undensified aggregates

$\phi_{a g g, \infty}$ solids fraction within fully densified aggregates

$\phi_{\infty}$ operating solids fraction of a fully densified thickener

$\phi_{f}$ feed solids fraction

$\phi_{u}$ underflow solids fraction 


\section{References}

Arbuthnot, I., Belke, J. V., R., T., Belke, J., Belke, Y. V., 2010. Separation device e.g. thickener for separating ground ore from water in e.g. mineral processing field, has shearing device that is moved in different direction with respect to rotation direction of rake assembly. Patent WO2010031119-A1, Outotec Oyj.

Batchelor, G. K., 1967. An Introduction to Fluid Dynamics, 1st Edition. Cambridge University Press, Cambridge.

Berres, S., Bürger, R., Coronel, A., Sepulveda, M., 2005a. Numerical identification of parameters for a flocculated suspension from centrifugation measurements during batch centrifugation. Chem. Engng J. 111 (2-3), 91-103, Special Issue. ECI Conference on Solid-Liquid Separation Systems IV - Solid-Liquid Separation Systems IV 03.

Berres, S., Burger, R., Coronel, A., Sepulveda, M., 2005b. Numerical identification of parameters for a strongly degenerate convection-diffusion problem modelling centrifugation of flocculated suspensions. Appl. Num. Math. 52, 311-337.

Boger, D. V., 2009. Rheology and the resource industries. Chem. Engng Sci. 64, 45254536.

Bürger, R., Bustos, M. C., Concha, F., 1999. Settling velocities of particulate systems: 9. Phenomenological theory of sedimentation processes: Numerical simulation of the transient behavior of flocculated suspensions in an ideal batch or continuous thickener. Int. J. Miner. Processing 55 (4), 267-282.

Bürger, R., Concha, F., 1998. Mathematical model and numerical simulation of the settling of flocculated suspensions. Int. J. Multiphase Flow 24, 1005-1023.

Buscall, R., White, L. R., 1987. The consolidation of concentrated suspensions. J. Chem. Soc. - Faraday Trans. I 83, 873-891.

Concha, F., Bustos, M. C., 1991. Settling velocities of particulate systems. 6. Kynch sedimentation processes: Batch settling. Int. J. Miner. Processing 32, 193-212. 
Concha, F., Bustos, M. C., 1992. Settling velocities of particulate systems. 7. Kynch sedimentation processes: Continuous thickening. Int. J. Miner. Processing 34, 33-51.

de Kretser, R. G., Usher, S. P., Scales, P. J., Boger, D. V., Landman, K. A., 2001. Rapid filtration measurement of dewatering design and optimisation parameters. AIChE. J. 47, 1758-1769.

Dickinson, J. E., Galvin, K. P., 2013. Fluidized bed desliming in fine particle flotationPart I. Accepted for publication by Chem. Engng. Sci. doi: 10.1016/j.ces.2013.11.006.

Doucet, J., Paradis, R., 2010. Thickening/mud stacking technology - an environmental approach to residue management. In: Paste 2010: Proceedings of the 13th International Seminar on Paste and Thickened Tailings, Toronto, ON, 3-6 May. Published by Australian Centre for Geomechanics, Nedlands, WA, pp. 3-21.

Fitch, B., 1966. Current theory and thickener design. Ind. Eng. Chem. 58 (10), 18-28.

Gladman, B., Usher, S. P., Scales, P. J., 2006. Compressive rheology of aggregated particulate suspensions. Korea-Australia Rheol. J. 18 (4), 191-197.

Gladman, B. R., 2006. The effect of shear on dewatering of flocculated suspensions. PhD thesis, University of Melbourne.

Gladman, B. R., de Kretser, R. G., Rudman, M., Scales, P. J., 2005. Effect of shear on particulate suspension dewatering. Chem. Eng. Res. Des. 83, 933-936.

Gladman, B. R., Rudman, M., Scales, P. J., 2010. The effect of shear on gravity thickening: Pilot scale modelling. Chem. Engng Sci. 65, 4293-4301.

Green, M. D., Landman, K. A., de Kretser, R. G., Boger, D. V., 1998. Pressure filtration technique for complete characterization of consolidating suspensions. Ind. Eng. Chem. Res. 37, 4152-4156.

Jones, H., Boger, D. V., 2012. Sustainability and waste management in the resource industries. Ind. Eng. Chem. Res. 51, 10057-10065. 
Kynch, G. J., 1952. A theory of sedimentation. Trans. Faraday Soc. 48, 166-176.

Landman, K. A., Sirakoff, C., White, L. R., 1991. Dewatering of flocculated suspensions by pressure filtration. Phys. Fluids A 3, 1495-1509.

Landman, K. A., White, L. R., 1992. Determination of the hindered settling factor for flocculated suspensions. AIChE. J. 38, 184-192.

Landman, K. A., White, L. R., 1994. Solid/liquid separation of flocculated suspensions. Adv. Colloid Interface Sci. 51, 175-246.

Landman, K. A., White, L. R., Buscall, R., 1988. The continuous-flow gravity thickener: Steady state behavior. AIChE. J. 34, 239-252.

Lester, D. R., Usher, S. P., Scales, P. J., 2005. Estimation of the hindered settling function $R(\phi)$ from batch-settling tests. AIChE. J. 51, 1158-1168.

Loan, C., Arbuthnot, I., 2010a. Innovative technology for optimised thickening sedimentation. In: XXV International Minerals Processing Congress - IMPC 2010, Smarter Processing for the Future, Brisbane, QLD, 6-10 Sep. Published by AusIMM, Carlton, VIC, pp. 3663-3674.

Loan, C., Arbuthnot, I., 2010b. Transforming paste thickener technology. In: Paste 2010: Proceedings of the 13th International Seminar on Paste and Thickened Tailings, Toronto, ON, 3-6 May. Published by Australian Centre for Geomechanics, Nedlands, WA, pp. 365-374.

Martin, A. D., 2004a. Filtration of flocculated suspensions under declining pressure. AIChE. J. 50, 1418-1430.

Martin, A. D., 2004b. Optimisation of clarifier-thickeners processing stable suspensions for turn-up/turn-down. Water Res. 38, 1568-1578.

Mills, P. D. A., Goodwin, J. W., Grover, B. W., 1991. Shear field modification of strongly flocculated suspensions - Aggregate morphology. Colloid \& Polym. Sci. 269, 949-963. 
Moncrieff, A. G., 1964. Theory of thickener design based on batch sedimentation tests. Trans. Institution Mining \& Metallurgy 73, 729-759.

Press, W. H., Teukolsky, S. A., Vetterling, W. T., Flannery, B. P., 1992. Numerical Recipes in C: The Art of Scientific Computing, 2nd Edition. Cambridge University Press, Cambridge.

Rudman, M., Simic, K., Paterson, D. A., Strode, P., Brent, A., Sutalo, I. D., 2008. Raking in gravity thickeners. Int. J. Miner. Processing 86, 114-130.

Schoenbrunn, F., 2011. Dewatering to higher densities - An industry review. In: Paste 2011: Proceedings of the 14th International Seminar on Paste and Thickened Tailings, Perth, WA, 5-7 Apr. Published by Australian Centre for Geomechanics, Nedlands, WA, pp. 19-23.

Shannon, P. T., Stroupe, E., Tory, E. M., 1963. Batch and continuous thickening. Basic theory: Solids flux for rigid spheres. Ind. Eng. Chem. Fundamen. 2, 203-211.

Shannon, P. T., Tory, E. M., 1965. Settling of slurries: New light on an old operation. Ind. Eng. Chem. 57 (2), 18-25.

Shannon, P. T., Tory, E. M., DeHaas, R. D., Stroupe, E. P., 1964. Batch and continuous thickening: Prediction of batch settling behavior from initial rate data with results for rigid spheres. Ind. Eng. Chem. Fundamen. 3, 250-260.

Stickland, A. D., Burgess, C., Dixon, D. R., Harbour, P. J., Scales, P. J., Studer, L. J., Usher, S. P., 2008. Fundamental dewatering properties of wastewater treatment sludge from filtration and sedimentation testing. Chem. Engng Sci. 63, 5283-5290.

Stickland, A. D., White, L. R., Scales, P. J., 2006. Modeling of solid-bowl batch centrifugation of flocculated suspensions. AIChE. J. 52, 1351-1362.

Talmage, W. P., Fitch, E. B., 1955. Determining thickener unit areas. Ind. Eng. Chem. $47(1), 38-41$. 
Usher, S. P., Scales, P. J., 2005. Steady state thickener modelling from the compressive yield stress and hindered settling function. Chem. Engng J. 111, 253-261.

Usher, S. P., Spehar, R., Scales, P. J., 2009. Theoretical analysis of aggregate densification: Impact on thickener performance. Chem. Engng J. 151, 202-208.

Usher, S. P., Studer, L. J., Wall, R. C., Scales, P. J., 2013. Characterisation of dewaterability from equilibrium and transient centrifugation test data. Chem. Engng. Sci. 93, $277-291$.

van Deventer, B. B. G., 2012. Aggregate densification behaviour in sheared suspensions. PhD thesis, University of Melbourne.

van Deventer, B. B. G., Usher, S. P., Kumar, A., Rudman, M., Scales, P. J., 2011. Aggregate densification and batch settling. Chem. Engng J. 171, 141-151.

Zhang, Y., Grassia, P., Martin, A. D., 2013a. Mathematical modelling of time-dependent densified thickeners. Chem. Engng Sci. 99, 103-112.

Zhang, Y., Grassia, P., Martin, A. D., 2013b. Prediction of thickener performance with aggregate densification. Chem. Engng Sci. 101, 346-358. 


\begin{tabular}{rrr}
\hline \multicolumn{1}{c}{$Q=0.25$} & $l$ \\
$T_{\text {res }}$ & & 0.0188 \\
\hline$\frac{1}{2}$ & & 0.0404 \\
1 & & 0.0832 \\
2 & & 0.1568 \\
4 & & $l$ \\
\hline & $Q=0.1$ & \\
$T_{\text {res }}$ & $T_{\text {pre }}$ & \\
\hline$\frac{1}{2}$ & 0.0459 & 0.0984 \\
1 & 0.3049 & 0.1498 \\
2 & 0.6449 & 0.2823 \\
4 & 0.8727 & 0.5928 \\
\hline
\end{tabular}

Table 1: Computed dimensionless settling zone heights $l$ vs dimensionless solids residence time in the shear field $T_{\text {res }}$ for suspension flux $Q=0.025$ and $Q=0.1$. In the case $Q=0.1$, preshearing occurs before entering the settling zone: the dimensionless preshear time $T_{p r e}$ is also reported. For 'typical' parameter values presented in the appendix, one dimensionless flux unit corresponds to $10^{-2} \mathrm{~m} \mathrm{~s}^{-1}$, one dimensionless time unit corresponds to $500 \mathrm{~s}$, and one dimensionless height unit corresponds to $5 \mathrm{~m}$. 

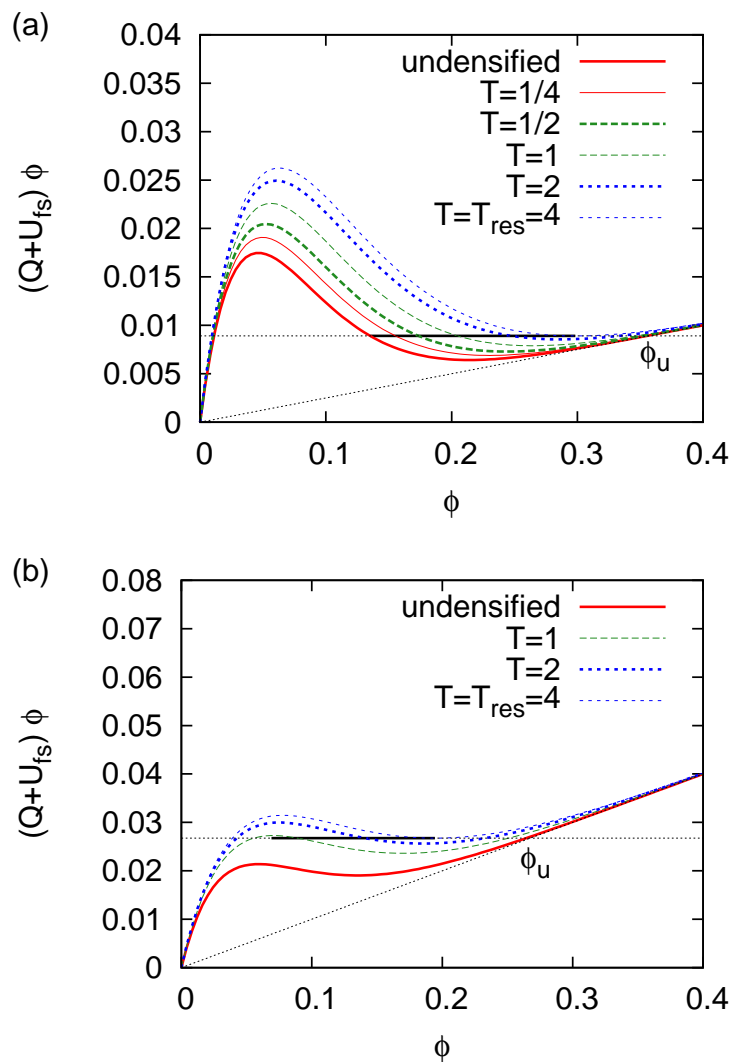

Figure 1: Solids flux $Q_{s} \equiv\left(Q+U_{f s}\right) \phi$ vs solids fraction $\phi$ at various different densification times $T$ up to a total residence time $T_{\text {res }}=4$, (a): small suspension flux $Q=0.025$; (b): comparatively large suspension flux $Q=0.10$. For comparison, the critical suspension flux at which the solids flux loses its local minimum with respect to solids fraction (in the undensified case) is $Q_{c r i t, 0}=0.148$. The range of solids fractions encountered in the thickener settling zone is indicated by the solid horizontal line (specifically where the flux curve for each $T$ value intersects that line), whilst the underflow solids fraction $\phi_{u}$ is also labelled. 


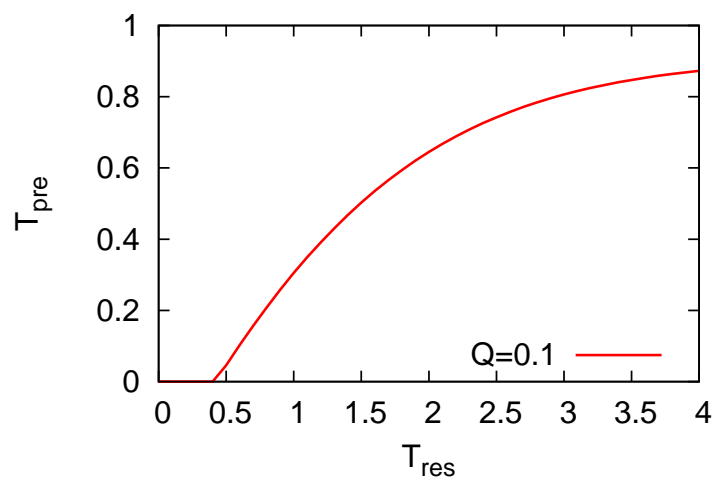

Figure 2: The preshearing time $T_{p r e}$ as a function of the residence time $T_{r e s}$ in the case where suspension flux $Q=0.1$. 


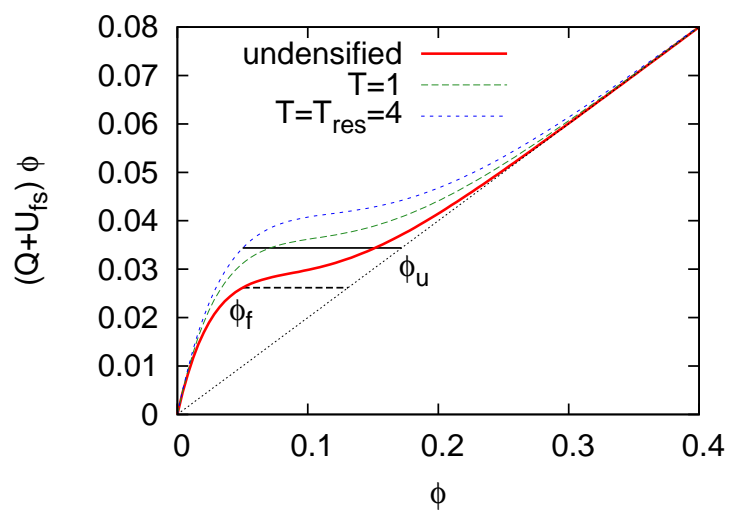

Figure 3: Solids flux $Q_{s} \equiv\left(Q+U_{f s}\right) \phi$ vs solids fraction $\phi$ for a system with residence time $T_{\text {res }}=4$, at various densification times $T$, specifically $T \rightarrow 0$ (the undensified state), $T=1$ and $T=T_{\text {res }}=4$. The suspension flux $Q$ is chosen sufficiently large $(Q=0.2)$ that the flux curves do not exhibit local minima. The feed solids fraction is chosen as $\phi_{f}=0.05$ and this determines the solids flux $\left(Q_{s}=0.0343\right.$ here with $T_{\text {res }}=4$ ). The underflow solids fraction $\phi_{u}$ is determined as the ratio $Q_{s} / Q$ (geometrically this is the right hand end of the solid horizontal segment shown on the graph; $\phi_{u}=0.171$ here). For undensified operation at the same feed solids fraction, a lesser solids flux $\left(Q_{s}=0.0261\right)$ and a correspondingly lesser underflow solids fraction (the right hand end of the dashed horizontal segment; $\phi_{u}=0.130$ ) are obtained. 

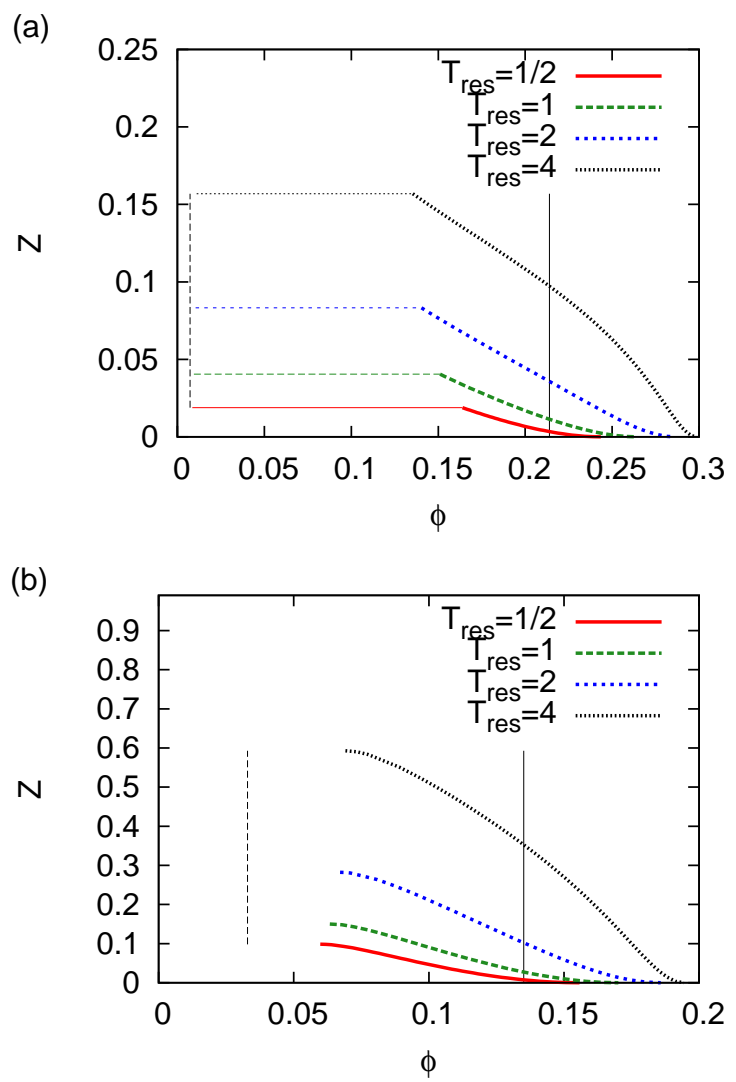

Figure 4: Profile of solids fraction $\phi$ vs dimensionless vertical coordinate $Z$ in the case where (a) suspension flux $Q=0.025$ and (b) suspension flux $Q=0.1$, for various residence times in the shear field $T_{\text {res }}=\frac{1}{2}, 1$, 2 and 4 . Note that for 'typical' parameter values presented in the appendix, one dimensionless height unit corresponds to $5 \mathrm{~m}$. In each case plotted, the $\phi$ vs $Z$ profile is indicated by a thick curve. However in (a) (albeit not (b)), it is permitted to have a jump in solids fraction from an exceedingly dilute value to the solids fraction corresponding to the top of the profile: these jumps are indicated by the thin horizontal lines extending from the top of each $\phi$ vs $Z$ profile. The vertical solid line (in both cases (a) and (b)) indicates the (uniform) solids fraction operating point that would be computed in an undensified system with the same suspension flux $Q$ (albeit a lower $Q_{s}$ ). With undensified operation, again a jump is possible from an exceedingly dilute state (the vertical dashed line in each case). 


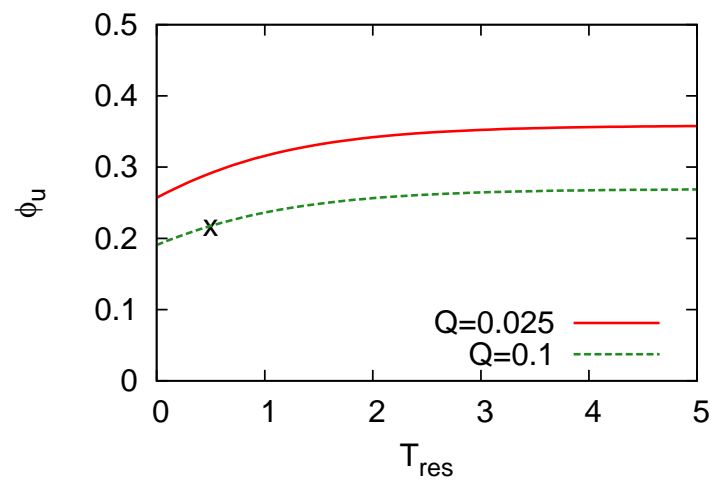

Figure 5: Underflow solids flux $\phi_{u}$ vs residence time $T_{r e s}$ in the shear field for two different suspension fluxes $Q=0.025$ and $Q=0.1$. The symbol $\times$ in the $Q=0.1$ case separates systems without preshear from those for which preshear occurs. 


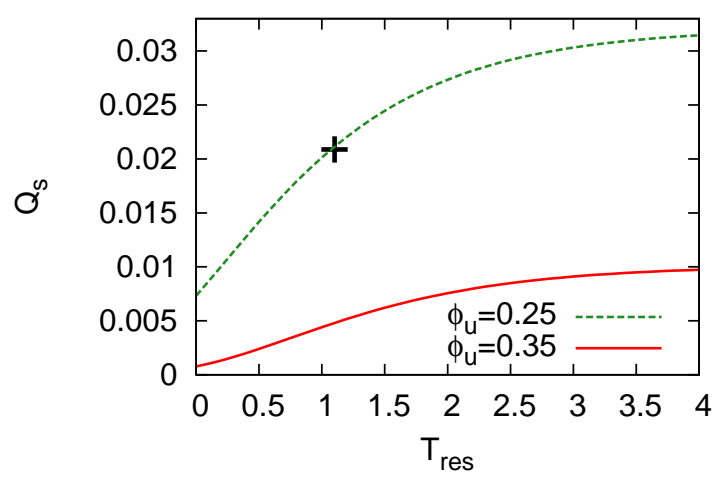

Figure 6: Solids flux $Q_{s}$ vs residence time $T_{r e s}$ in the shear field for two different underflow solids fractions $\phi_{u}=0.25$ and $\phi_{u}=0.35$. The symbol + in the $\phi_{u}=0.25$ case separates systems that do not involve preshear from those that do. 

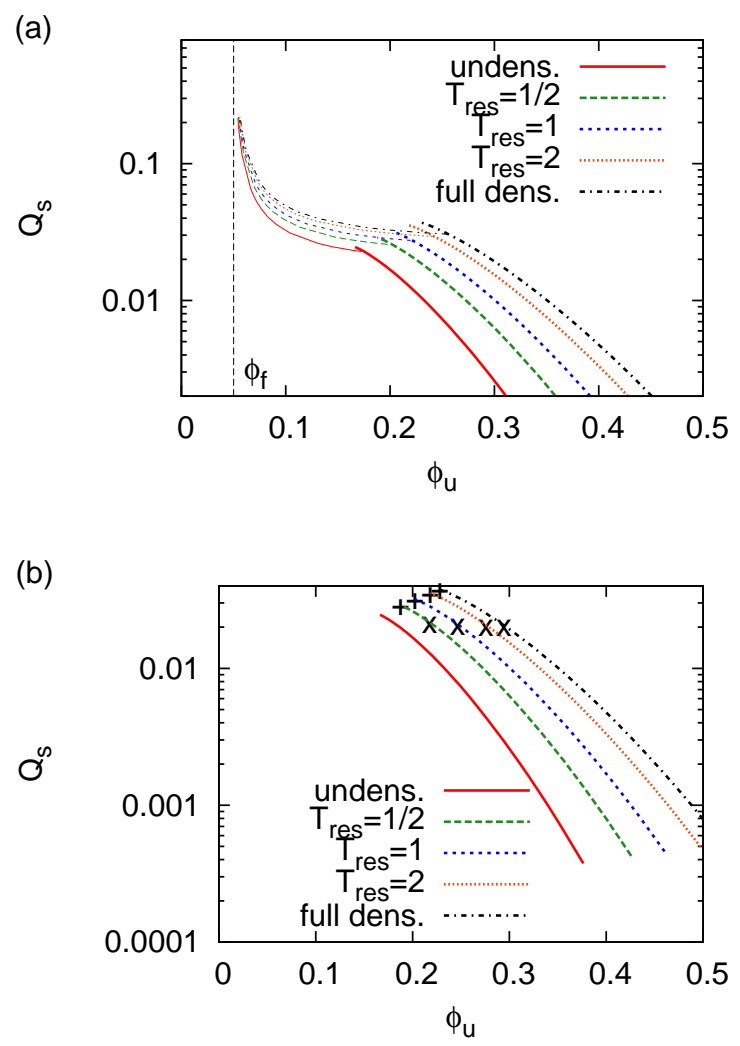

Figure 7: Predictions of the thickener model for solids flux $Q_{s}$ vs underflow solids fraction $\phi_{u}$. Data are shown for the undensified case and for the fully densified case, as well as at various (finite) residence times $T_{\text {res }}=\frac{1}{2}, 1$ and 2. (a) Two branches of solutions are shown: one at lower solids fluxes and higher underflow solids fractions (the situation for which the corresponding solids flux vs solids fraction curve has a local minimum and maximum, so that operation is constrained by the shape of the flux curve (Dickinson and Galvin, 2013)) and one at higher solids fluxes and lower solids fraction (the thickener operates at the feed solids fraction (taken to be $\phi_{f}=0.05$ here), but is not constrained by the shape of the flux curve at any other solids fraction). (b) Detailed view of the constrained branch. The symbols $\times$ separate systems without preshear from those for which preshear occurs. Meanwhile the symbols + indicate the limit at which the solids flux curve ceases to have a local minimum and maximum. In order to benefit from aggregate densification at these latter points, all the aggregate densification must take place in the form of preshear, with no further aggregate densification in the thickener itself. 

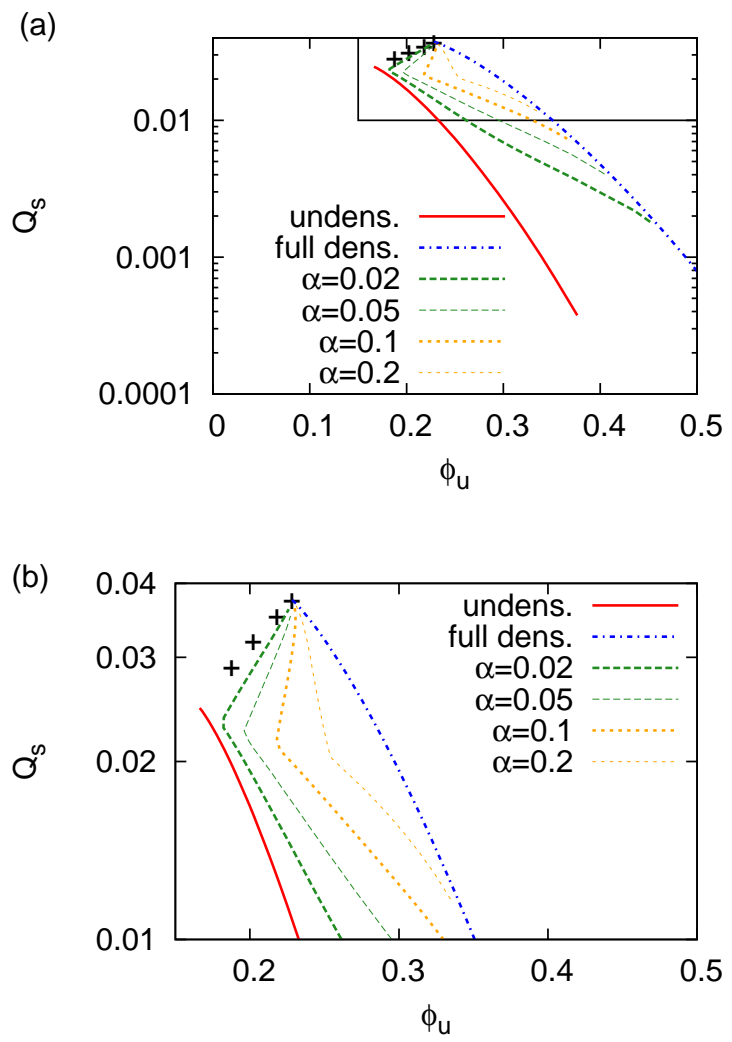

Figure 8: (a) Predictions of the thickener model for dimensionless solids flux $Q_{s}$ vs underflow solids fraction $\phi_{u}$ for the undensified case and for the fully densified case, as well as at various values of the dimensionless densification rate parameter $\alpha(\alpha=0.02,0.05,0.1$ and 0.2$)$. Note that physically, different $\alpha$ values correspond to varying the densification rate (at fixed height of the settling zone) or else to varying the height of the settling zone (at fixed densification rate): for 'typical' parameter values presented in the appendix, $\alpha=0.02$ corresponds to a zone height $0.1 \mathrm{~m}$, whilst $\alpha=0.2$ corresponds to a zone height $1 \mathrm{~m}$. The symbols + correspond to the points at which the solids flux curve ceases to have a local minimum and maximum. At these points, all the aggregate densification is realised via preshear, with no additional aggregate densification occurring in the thickener itself. As a result, these points (like the undensified case itself) correspond formally to the limit $\alpha \rightarrow 0$. (b) A zoomed view of the upper right hand corner of the upper figure. 